\title{
Homogenization Problem in a Domain with Double Oscillating Boundary
}

\author{
Jie Zhao $\mathbb{D}$ and Juan Wang \\ College of Science, Zhongyuan University of Technology, Zhengzhou 450007, China \\ Correspondence should be addressed to Jie Zhao; kaifengajie@163.com
}

Received 25 March 2018; Accepted 16 September 2018; Published 21 October 2018

Academic Editor: Emilio Turco

Copyright ( 2018 Jie Zhao and Juan Wang. This is an open access article distributed under the Creative Commons Attribution License, which permits unrestricted use, distribution, and reproduction in any medium, provided the original work is properly cited.

In this paper, we study the convergence of solutions for homogenization problems about the Poisson equation in a domain with double oscillating locally periodic boundary. Such a problem arises in the processing of devices with very small features. We utilize second-order Taylor expansion of boundary data in combination with boundary correctors to obtain the convergence rate in $H^{1}$ norm. This work explores the domain with double oscillating boundary and also shows the influence of the amplitudes and periods of the oscillations to convergence rates of solutions.

\section{Introduction}

Several important problems arising in physics and engineering lead to considering boundary value problems in domains with oscillating boundaries. Such problems arise in the context of fluid flows over a rough surface [1,2], of reinforcement by a thin layer [3], or of electromagnetic scattering by an obstacle with a periodic coating $[4,5]$. Indeed, the oscillating boundary results can be applied to the homogenization of neutronic diffusion or transport equation [6-8]. Studying the oscillating boundary is also the key for determining interface transmission conditions in various mechanical problems [9$11]$.

In this paper, we are interested in the following boundary value problem for the Poisson equation in a domain with double oscillating locally periodic boundary:

$$
\begin{aligned}
-\Delta u_{\varepsilon} & =f(x) \text { in } \Omega_{\varepsilon}, \\
\frac{\partial u_{\varepsilon}}{\partial \nu_{\varepsilon}}+p\left(x^{\prime}, \frac{x^{\prime}}{\varepsilon^{\alpha}}\right) u_{\varepsilon} & =g\left(x^{\prime}, \frac{x^{\prime}}{\varepsilon^{\alpha}}\right) \quad \text { on } \Gamma_{1}^{\varepsilon}, \\
\frac{\partial u_{\varepsilon}}{\partial \nu_{\varepsilon}}+q\left(x^{\prime}, \frac{x^{\prime}}{\varepsilon^{\beta}}\right) u_{\varepsilon} & =h\left(x^{\prime}, \frac{x^{\prime}}{\varepsilon^{\beta}}\right) \quad \text { on } \Gamma_{2}^{\varepsilon}, \\
u_{\varepsilon} & =0 \text { on } \Gamma_{3} .
\end{aligned}
$$

where $x^{\prime}=\left(x_{1}, x_{2} \cdots, x_{n-1}\right) \in(0,1)^{n-1}$ and $v_{\varepsilon}$ is the outward unit normal. The domain $\Omega_{\varepsilon}$ is a bounded domain in $\mathbb{R}^{n}$ and $\partial \Omega_{\varepsilon}=\Gamma_{1}^{\mathcal{E}} \cup \Gamma_{2}^{\mathcal{E}} \cup \Gamma_{3}$, where hypersurfaces

$$
\Gamma_{1}^{\varepsilon}: x_{n}=\varepsilon^{\alpha} S\left(x^{\prime}, \frac{x^{\prime}}{\varepsilon^{\alpha}}\right), \quad \alpha>0,
$$

and

$$
\Gamma_{2}^{\varepsilon}: x_{n}=1-\varepsilon^{\beta} T\left(x^{\prime}, \frac{x^{\prime}}{\varepsilon^{\beta}}\right), \quad \beta>0 .
$$

Without loss of generality, we assume that $\Omega_{\varepsilon}$ is a domain bounded by hyperplanes $\left\{x_{i}=0\right\}$ and $\left\{x_{i}=1\right\}(i=1,2 \cdots n-$ 1) and hypersurfaces $\Gamma_{1}^{\varepsilon}$ and $\Gamma_{2}^{\varepsilon}$.

We suppose that

$$
\begin{aligned}
& p\left(x^{\prime}, \xi^{\prime}\right) \geq 0, \\
& q\left(x^{\prime}, \eta^{\prime}\right) \geq 0, \\
& S\left(x^{\prime}, \xi^{\prime}\right) \geq 0, \\
& T\left(x^{\prime}, \eta^{\prime}\right) \geq 0,
\end{aligned}
$$

$$
\text { where } \xi^{\prime}=\frac{x^{\prime}}{\varepsilon^{\alpha}} \text { and } \eta^{\prime}=\frac{x^{\prime}}{\varepsilon^{\beta}} .
$$


The conditions $p\left(x^{\prime}, \xi^{\prime}\right) \geq 0$ and $q\left(x^{\prime}, \eta^{\prime}\right) \geq 0$ ensure the existence and uniqueness of solution for problem (1). This can be guaranteed by Lax-Milgram Theorem. The assumptions $S\left(x^{\prime}, \xi^{\prime}\right) \geq 0$ and $T\left(x^{\prime}, \eta^{\prime}\right) \geq 0$ ensure that $0 \leq x_{n} \leq 1$. This makes the proof a bit more simpler. In fact, all the results of this paper remain valid even without the nonnegativity of $S\left(x^{\prime}, \xi^{\prime}\right)$ and $T\left(x^{\prime}, \eta^{\prime}\right)$.

We also impose the smoothness condition and periodicity condition

$$
\begin{aligned}
& p\left(x^{\prime}, \xi^{\prime}\right), g\left(x^{\prime}, \xi^{\prime}\right) \text { and } S\left(x^{\prime}, \xi^{\prime}\right) \\
& \text { are smooth functions in }\left(x^{\prime}, \xi^{\prime}\right) \text { and } 1 \\
& \text { - periodic in } \xi^{\prime}, \\
& q\left(x^{\prime}, \eta^{\prime}\right), h\left(x^{\prime}, \eta^{\prime}\right) \text { and } T\left(x^{\prime}, \eta^{\prime}\right) \\
& \text { are smooth functions in }\left(x^{\prime}, \eta^{\prime}\right) \text { and } 1 \\
& \text { - periodic in } \eta^{\prime} .
\end{aligned}
$$

Here we do not aim to obtain the optimal smoothness but rather focus on the method itself.

In order to avoid technical difficulties, we assume that

$$
\begin{aligned}
& g\left(x^{\prime}, \xi^{\prime}\right) \equiv 0, \\
& h\left(x^{\prime}, \eta^{\prime}\right) \equiv 0, \\
& S\left(x^{\prime}, \xi^{\prime}\right) \equiv 0, \\
& T\left(x^{\prime}, \eta^{\prime}\right) \equiv 0, \\
& \quad \text { on } x_{i}=0 \text { and } x_{i}=1,
\end{aligned}
$$

where $i=1,2 \cdots n-1$. The conditions on $g$ and $h$ are compatibility conditions. The assumptions about $S$ and $T$ are needed for dealing with the situation when the oscillating boundary intersects the flat boundary.

As the authors are aware, there are many papers about results of convergence rates for elliptic homogenization problems with oscillating boundary data. In 1997, A. Friedman, B. $\mathrm{Hu}$, and Y. Liu [12] studied two-dimensional domain, whose boundary is oscillating according to three scales. They extended the results of Belyaev $[13,14]$ to the three-scale oscillating boundary. In 1999, G. A. Chechkin, A. Friedman, and A. L. Piatnitski [15] considered such problem including some parameters and obtained some error estimates in $H^{1}$ norm. Their [15] method is following a general procedure in homogenization but without using correctors.

Recently, there has been a surge of activity in the theory of homogenization in domain with oscillating boundary data. In 2012, D. Gérard and N. Masmoudi [16] studied the homogenization of elliptic system with Dirichlet boundary conditions, when the coefficients of the system and the boundary data are $\varepsilon$-periodic. They obtained the solutions convergence in $L^{2}$ with a power rate in $\varepsilon$. In 2013, H. Aleksanyan, H. Shahgholian, and P. Sjölin [17] studied the the boundary value homogenization for Dirichlet problem. In particular, they proved pointwise and $L^{p}$ convergence results. Their method is based on analysis of oscillatory integrals. The papers [18, 19] were devoted to the investigations of the homogenization problem for the Poisson equation in a thin domain with an oscillating boundary.

For such domain with rapidly oscillating boundary, there are many directions enabling us to consider. Boundary-value problems involving rapidly oscillating boundaries or interfaces appear in many fields of physics and engineering sciences, and it will be interesting to investigate the asymptotic behavior of the solutions of spectral problems. Y. Amirat, G. A. Chechkin, and R. R. Gadyl'shin were devoted to prove the $H^{1}$ convergence of the eigenvalues and eigenfunctions to the eigenvalues and eigenfunctions of the homogenization problem, via the traditional method of asymptotic expansions. Moreover, it is worth noticing that many mathematical works have been contributed to the asymptotic analysis of problems in domains with random microstructure. For instance, in 2011, Y. Amirat and other scholars, after adding some extra assumptions on the random variables, were able to obtain the convergence results of solutions in $H^{1}$. This is also an interesting problem.

One may consult several outstanding sources [20-26] for background and overview of the homogenization theory.

The main difference of the present work in relation to previous existing work is that this work explores the domain with double oscillating boundary and also shows the influence of the amplitudes and periods of the oscillations to convergence rates of solutions. Meanwhile, with different traditional asymptotic expansions method, this paper improves the convergence rate results in $H^{1}$-norm by virtue of boundary correctors that can be used to obtain effective approximation.

We now describe the outline of this paper. Section 2 contains some basic formulas and estimates which are important to obtain error estimates. In Section 3, we show that the solution $u_{\varepsilon}$ of problem (1) converges to the solution $u_{0}$ of the corresponding homogenized problem in the $H^{1}$-norm with error estimate up to order of $O\left(\varepsilon^{\alpha / 2}+\varepsilon^{\beta / 2}\right)$. In Section 4 , we construct correctors that play important role in improving the power in $\varepsilon$. Next, we improve the error estimate up to order of $O\left(\varepsilon^{\alpha}+\varepsilon^{\beta}\right)$ in Section 5 . This can be obtained via the correctors. Following the same line of research, in Section 6, we obtain an approximation to $u_{\varepsilon}$ with error estimate up to order of $\left(\varepsilon^{3 \alpha / 2}+\varepsilon^{3 \beta / 2}\right)$ in $H^{1}$-norm by using correctors and second-order Taylor's expansion.

\section{Preliminaries}

We consider the homogenized problem associated with problem (1) in the form

$$
\begin{aligned}
-\Delta u_{0} & =f(x) \quad \text { in } \Omega_{0}, \\
\frac{\partial u_{0}}{\partial v_{0}}+P\left(x^{\prime}\right) u_{0} & =G\left(x^{\prime}\right) \quad \text { on } \Gamma_{1},
\end{aligned}
$$




$$
\begin{aligned}
\frac{\partial u_{0}}{\partial \nu_{0}}+Q\left(x^{\prime}\right) u_{0} & =H\left(x^{\prime}\right) \quad \text { on } \Gamma_{2}, \\
u_{0} & =0 \text { on } \Gamma_{3},
\end{aligned}
$$

where $\Omega_{0} \subset(0,1)^{n}$ and $v_{0}$ is the outward unit normal. Here $\Gamma_{1}=\left\{x^{\prime} \in[0,1]^{n-1}, x_{n}=0\right\}$ and $\Gamma_{2}=\left\{x^{\prime} \in[0,1]^{n-1}, x_{n}=\right.$ $1\}$. Functions $P\left(x^{\prime}\right), Q\left(x^{\prime}\right), G\left(x^{\prime}\right)$, and $H\left(x^{\prime}\right)$ are defined as follows:

$$
\begin{aligned}
& P\left(x^{\prime}\right) \\
& \quad \int_{0}^{1} \ldots \int_{0}^{1}\left[1+\left|\nabla_{\xi^{\prime}} S\left(x^{\prime}, \xi^{\prime}\right)\right|^{2}\right]^{1 / 2} p\left(x^{\prime}, \xi^{\prime}\right) d \xi^{\prime}, \\
& G\left(x^{\prime}\right)=\int_{0}^{1} \ldots \int_{0}^{1}\left[1+\left|\nabla_{\xi^{\prime}} S\left(x^{\prime}, \xi^{\prime}\right)\right|^{2}\right]^{1 / 2} g\left(x^{\prime}, \xi^{\prime}\right) d \xi^{\prime}, \\
& Q\left(x^{\prime}\right) \\
& \quad=\int_{0}^{1} \ldots \int_{0}^{1}\left[1+\left|\nabla_{\eta^{\prime}} T\left(x^{\prime}, \eta^{\prime}\right)\right|^{2}\right]^{1 / 2} q\left(x^{\prime}, \eta^{\prime}\right) d \eta^{\prime}, \\
& H\left(x^{\prime}\right) \\
& \quad=\int_{0}^{1} \ldots \int_{0}^{1}\left[1+\left|\nabla_{\eta^{\prime}} T\left(x^{\prime}, \eta^{\prime}\right)\right|^{2}\right]^{1 / 2} h\left(x^{\prime}, \eta^{\prime}\right) d \eta^{\prime} .
\end{aligned}
$$

As a preliminary step, we shall prove some propositions.

Proposition 1. There exists a constant $C$ independent of $\varepsilon$ such that, for any $v \in H^{1}\left(\Omega_{0}\right)$, the following inequalities

$$
\begin{aligned}
& \left\|v\left(x^{\prime}, \varepsilon^{\alpha} S\left(x^{\prime}, \frac{x^{\prime}}{\varepsilon^{\alpha}}\right)\right)-v\left(x^{\prime}, 0\right)\right\|_{L^{2}\left(\Gamma_{1}\right)} \\
& \quad \leq C \varepsilon^{\alpha / 2}\|v\|_{H^{1}\left(\Omega_{0}\right)}
\end{aligned}
$$

and

$$
\begin{aligned}
& \left\|v\left(x^{\prime}, 1-\varepsilon^{\beta} T\left(x^{\prime}, \frac{x^{\prime}}{\varepsilon^{\beta}}\right)\right)-v\left(x^{\prime}, 1\right)\right\|_{L^{2}\left(\Gamma_{2}\right)} \\
& \quad \leq C \varepsilon^{\beta / 2}\|v\|_{H^{1}\left(\Omega_{0}\right)}
\end{aligned}
$$

hold.

Proof. Without loss of generality, we may assume $v \in$ $C^{\infty}\left(\Omega_{0}\right)$. A simple calculation then gives

$$
\begin{gathered}
v\left(x^{\prime}, \varepsilon^{\alpha} S\left(x^{\prime}, \frac{x^{\prime}}{\varepsilon^{\alpha}}\right)\right)-v\left(x^{\prime}, 0\right) \\
=\int_{0}^{\varepsilon^{\alpha} S\left(x^{\prime}, x^{\prime} / \varepsilon^{\alpha}\right)} \frac{\partial v\left(x^{\prime}, t\right)}{\partial t} d t .
\end{gathered}
$$

It follows from Hölder's inequality that

$$
\begin{aligned}
& \left|v\left(x^{\prime}, \varepsilon^{\alpha} S\left(x^{\prime}, \frac{x^{\prime}}{\varepsilon^{\alpha}}\right)\right)-v\left(x^{\prime}, 0\right)\right|^{2} \\
& \leq C \varepsilon^{\alpha} \int_{0}^{\varepsilon^{\alpha} S\left(x^{\prime}, x^{\prime} / \varepsilon^{\alpha}\right)}\left|\frac{\partial v\left(x^{\prime}, t\right)}{\partial t}\right|^{2} d t .
\end{aligned}
$$

Integrating over $\Gamma_{1}$, we obtain (9). The proof of (10) is similar to (9). This completes the proof.

Proposition 2. There exists a constant $C$ independent of $\varepsilon$ such that, for any $v \in H^{1}\left(\Omega_{0}\right)$, the following estimate

$$
\|v\|_{L^{2}\left(\Omega_{0} \backslash \Omega_{\varepsilon}\right)} \leq C\left(\varepsilon^{\alpha / 2}+\varepsilon^{\beta / 2}\right)\|v\|_{H^{1}\left(\Omega_{0}\right)}
$$

is valid.

Proof. Note that

$$
v\left(x^{\prime}, 1\right)-v\left(x^{\prime}, \tau\right)=\int_{\tau}^{1} \frac{\partial v\left(x^{\prime}, t\right)}{\partial t} d t
$$

where $\tau \in\left[1-\varepsilon^{\beta} T\left(x^{\prime}, x^{\prime} / \varepsilon^{\beta}\right), 1\right]$.

It is easy to see that

$$
v^{2}\left(x^{\prime}, \tau\right) \leq 2 v^{2}\left(x^{\prime}, 1\right)+2(1-\tau) \int_{\tau}^{1}\left|\frac{\partial v\left(x^{\prime}, t\right)}{\partial t}\right|^{2} d t .
$$

Let $\tau=x_{n, 2}=1-\varepsilon^{\beta} T\left(x^{\prime}, x^{\prime} / \varepsilon^{\beta}\right)$, and, integrating over $(0,1)^{n-1}$, we obtain

$$
\begin{aligned}
\int_{0}^{1} \cdots \int_{0}^{1} \int_{x_{n, 2}}^{1} v^{2}\left(x^{\prime}, x_{n, 2}\right) d x \\
\leq 2 \varepsilon^{\beta} \max T\|v\|_{L^{2}(0,1)^{n-1}} \\
\quad+2 \varepsilon^{2 \beta} \max T^{2} \int_{0}^{1} \cdots \int_{0}^{1} \int_{x_{n, 2}}^{1}\left|\frac{\partial v\left(x^{\prime}, t\right)}{\partial t}\right|^{2} d t .
\end{aligned}
$$

A similar computation yields the following result:

$$
\begin{aligned}
& \int_{0}^{1} \cdots \int_{0}^{1} \int_{0}^{x_{n, 1}} v^{2}\left(x^{\prime}, x_{n, 1}\right) d x \\
& \leq 2 \varepsilon^{\alpha} \max S\|v\|_{L^{2}(0,1)^{n-1}}
\end{aligned}
$$

$$
+2 \varepsilon^{2 \alpha} \max S^{2} \int_{0}^{1} \cdots \int_{0}^{1} \int_{0}^{x_{n, 1}}\left|\frac{\partial v\left(x^{\prime}, t\right)}{\partial t}\right|^{2} d t,
$$

where $x_{n, 1}=\varepsilon^{\alpha} S\left(x^{\prime}, x^{\prime} / \varepsilon^{\alpha}\right)$.

Combining these two terms, we obtain (13). This completes the proof.

Proposition 3. Let $m\left(x^{\prime}, \xi^{\prime}\right), n\left(x^{\prime}, \eta^{\prime}\right)$ be 1-periodic in $\xi^{\prime}, \eta^{\prime}$ and satisfy

$$
\begin{aligned}
& \int_{0}^{1} \cdots \int_{0}^{1} m\left(x^{\prime}, \xi^{\prime}\right) d \xi^{\prime}=0 \text { and } \\
& \int_{0}^{1} \cdots \int_{0}^{1} n\left(x^{\prime}, \eta^{\prime}\right) d \eta^{\prime}=0
\end{aligned}
$$

respectively. Then, these inequalities

$$
\begin{aligned}
&\left|\int_{\Gamma_{1}} m\left(x^{\prime}, \frac{x^{\prime}}{\varepsilon^{\alpha}}\right) v\left(x^{\prime}\right) d x^{\prime}\right| \leq C \varepsilon^{\alpha / 2}\|v\|_{H^{1 / 2}\left(\Gamma_{1}\right)} \text { and } \\
&\left|\int_{\Gamma_{2}} n\left(x^{\prime}, \frac{x^{\prime}}{\varepsilon^{\beta}}\right) v\left(x^{\prime}\right) d x^{\prime}\right| \leq C \varepsilon^{\beta / 2}\|v\|_{H^{1 / 2}\left(\Gamma_{2}\right)}
\end{aligned}
$$


Proof. This proposition has been proved by G. A. Chechkin, A. Friedman, and A. L. Piatnitski in [15].

Proposition 4. There exists a constant $C$ independent of $\varepsilon$ such that, for all $v \in H^{1}\left(\Omega_{0}\right)$, the following estimates

$$
\begin{aligned}
& \mid \int_{\Gamma_{1}^{\varepsilon}} g\left(x^{\prime}, \frac{x^{\prime}}{\varepsilon^{\alpha}}\right) v\left(x^{\prime}, \varepsilon^{\alpha} S\left(x^{\prime}, \frac{x^{\prime}}{\varepsilon^{\alpha}}\right)\right) d \sigma_{1} \\
& -\int_{\Gamma_{1}} G\left(x^{\prime}\right) v\left(x^{\prime}, 0\right) d x^{\prime} \mid \leq C \varepsilon^{\alpha / 2}\|v\|_{H^{1}\left(\Omega_{0}\right)}
\end{aligned}
$$

and

$$
\begin{array}{r}
\mid \int_{\Gamma_{2}^{\varepsilon}} h\left(x^{\prime}, \frac{x^{\prime}}{\varepsilon^{\beta}}\right) v\left(x^{\prime}, 1-\varepsilon^{\beta} T\left(x^{\prime}, \frac{x^{\prime}}{\mathcal{E}^{\beta}}\right)\right) d \sigma_{2} \\
-\int_{\Gamma_{2}} H\left(x^{\prime}\right) v\left(x^{\prime}, 1\right) d x^{\prime} \mid \leq C \varepsilon^{\beta / 2}\|v\|_{H^{1}\left(\Omega_{0}\right)} .
\end{array}
$$

take place.

Proof. A direct computation shows that

$$
\begin{aligned}
& \left|\int_{\Gamma_{1}^{\varepsilon}} g\left(x^{\prime}, \frac{x^{\prime}}{\varepsilon^{\alpha}}\right) v\left(x^{\prime}, \varepsilon^{\alpha} S\left(x^{\prime}, \frac{x^{\prime}}{\varepsilon^{\alpha}}\right)\right) d \sigma_{1}-\int_{\Gamma_{1}} G\left(x^{\prime}\right) v\left(x^{\prime}, 0\right) d x^{\prime}\right| \\
& \quad=\left|\int_{\Gamma_{1}} g\left(x^{\prime}, \frac{x^{\prime}}{\varepsilon^{\alpha}}\right) v\left(x^{\prime}, \varepsilon^{\alpha} S\left(x^{\prime}, \frac{x^{\prime}}{\varepsilon^{\alpha}}\right)\right)\left[1+\left|\nabla_{\xi^{\prime}} S\right|^{2}\right]^{1 / 2} d x^{\prime}-\int_{\Gamma_{1}} G\left(x^{\prime}\right) v\left(x^{\prime}, 0\right) d x^{\prime}\right|+O\left(\varepsilon^{\alpha}\right)\|v\|_{H^{1}\left(\Omega_{0}\right)} \\
& \quad \leq\left|\int_{\Gamma_{1}} g\left(x^{\prime}, \frac{x^{\prime}}{\varepsilon^{\alpha}}\right)\left(v\left(x^{\prime}, \varepsilon^{\alpha} S\left(x^{\prime}, \frac{x^{\prime}}{\varepsilon^{\alpha}}\right)\right)-v\left(x^{\prime}, 0\right)\right)\left[1+\left|\nabla_{\xi^{\prime}} S\right|^{2}\right]^{1 / 2} d x^{\prime}\right| \\
& \quad+\left|\int_{\Gamma_{1}} g\left(x^{\prime}, \frac{x^{\prime}}{\varepsilon^{\alpha}}\right) v\left(x^{\prime}, 0\right)\left[1+\left|\nabla_{\xi^{\prime}} S\right|^{2}\right]^{1 / 2} d x^{\prime}-\int_{\Gamma_{1}} G\left(x^{\prime}\right) v\left(x^{\prime}, 0\right) d x^{\prime}\right|+C \varepsilon^{\alpha}\|v\|_{H^{1}\left(\Omega_{0}\right)} .
\end{aligned}
$$

This, together with Propositions 1 and 3, yields estimate (20). Similarly, one can prove (21).
Proposition 5. There exists a constant $C$ independent of $\varepsilon$ such that, for all $v \in H^{1}\left(\Omega_{0}\right)$, the following inequalities

$$
\begin{aligned}
& \left|\int_{\Gamma_{1}^{\varepsilon}} p\left(x^{\prime}, \frac{x^{\prime}}{\varepsilon^{\alpha}}\right) u_{0}\left(x^{\prime}, \frac{x^{\prime}}{\varepsilon^{\alpha}}\right) v\left(x^{\prime}, \varepsilon^{\alpha} S\left(x^{\prime}, \frac{x^{\prime}}{\varepsilon^{\alpha}}\right)\right) d \sigma_{1}-\int_{\Gamma_{1}} P\left(x^{\prime}\right) u_{0}\left(x^{\prime}, 0\right) v\left(x^{\prime}, 0\right) d x^{\prime}\right| \\
& \quad \leq C \varepsilon^{\alpha / 2}\left\|u_{0}\right\|_{H^{1}\left(\Omega_{0}\right)}\|v\|_{H^{1}\left(\Omega_{0}\right)}
\end{aligned}
$$

and

$$
\begin{aligned}
& \left|\int_{\Gamma_{2}^{\varepsilon}} q\left(x^{\prime}, \frac{x^{\prime}}{\varepsilon^{\beta}}\right) u_{0}\left(x^{\prime}, \frac{x^{\prime}}{\varepsilon^{\beta}}\right) v\left(x^{\prime}, 1-\varepsilon^{\beta} T\left(x^{\prime}, \frac{x^{\prime}}{\varepsilon^{\beta}}\right)\right) d \sigma_{2}-\int_{\Gamma_{2}} Q\left(x^{\prime}\right) u_{0}\left(x^{\prime}, 1\right) v\left(x^{\prime}, 1\right) d x^{\prime}\right| \\
& \leq C \varepsilon^{\beta / 2}\left\|u_{0}\right\|_{H^{1}\left(\Omega_{0}\right)}\|v\|_{H^{1}\left(\Omega_{0}\right)} .
\end{aligned}
$$

hold true.

Proof. This proposition can be proved in the same way as Proposition 4.

\section{Error Estimate up to $O\left(\varepsilon^{\alpha / 2}+\varepsilon^{\beta / 2}\right)$}

In this section, we will prove the error estimate up to the order of $O\left(\varepsilon^{\alpha / 2}+\varepsilon^{\beta / 2}\right)$. Our main result is the following theorem.
Theorem 6 (let $f \in L^{2}\left(\Omega_{\varepsilon}\right)$ ). Assume that $u_{\varepsilon}$ is a solution of problem (1)-(3). Suppose that $p\left(x^{\prime}, \xi^{\prime}\right), g\left(x^{\prime}, \xi^{\prime}\right), q\left(x^{\prime}, \eta^{\prime}\right)$, $h\left(x^{\prime}, \eta^{\prime}\right), S\left(x^{\prime}, \xi^{\prime}\right)$, and $T\left(x^{\prime}, \eta^{\prime}\right)$ satisfy (4)-(6). Then there exists a constant $C$ which does not depend on $\varepsilon$, such that

$$
\left\|u_{\varepsilon}-u_{0}\right\|_{H^{1}\left(\Omega_{\varepsilon}\right)} \leq C\left(\varepsilon^{\alpha / 2}+\varepsilon^{\beta / 2}\right) \text {. }
$$

Remark 7. Following general procedure in homogenization, we introduce the associated bilinear form 


$$
B(u, v) \doteq \int_{\Omega_{\varepsilon}} \nabla u \cdot \nabla v d x+\int_{\Gamma_{1}^{\varepsilon}} p u v d \sigma_{1}+\int_{\Gamma_{2}^{\varepsilon}} q u v d \sigma_{2},
$$

where

$$
\begin{aligned}
d \sigma_{1} & =\left[1+\sum_{i=1}^{n-1}\left(\varepsilon^{\alpha} \frac{\partial S}{\partial x_{i}}+\frac{\partial S}{\partial \xi_{i}}\right)^{2}\right]^{1 / 2} d x^{\prime} \\
& =\left[1+\left|\nabla_{\xi^{\prime}} S\right|^{2}\right]^{1 / 2} d x^{\prime}+O\left(\varepsilon^{\alpha}\right) d x^{\prime}, \\
d \sigma_{2} & =\left[1+\sum_{i=1}^{n-1}\left(\varepsilon^{\beta} \frac{\partial T}{\partial x_{i}}+\frac{\partial T}{\partial \xi_{i}}\right)^{2}\right]^{1 / 2} d x^{\prime} \\
& =\left[1+\left|\nabla_{\eta^{\prime}} T\right|^{2}\right]^{1 / 2} d x^{\prime}+O\left(\varepsilon^{\beta}\right) d x^{\prime} .
\end{aligned}
$$

We shall establish

$$
B\left(u_{\varepsilon}-u_{0}, v\right) \leq C\left(\varepsilon^{\alpha / 2}+\varepsilon^{\beta / 2}\right)\|v\|_{H^{1}\left(\Omega_{0}\right)} .
$$

Then, we extend $u_{\varepsilon}-u_{0}$ into $\Omega_{0}$ without increasing the $H^{1}$ norm by more than a multiplicative constant. What remains is just to take $v=u_{\varepsilon}-u_{0}$ and use Poincaré's inequality. Then this theorem will be proved.

Proof. Since $\Omega_{\varepsilon} \subset \Omega_{0}$, every function $f \in L^{2}\left(\Omega_{\varepsilon}\right)$ can be easily extended by 0 in $\Omega_{0} \backslash \Omega_{\varepsilon}$ to become a function of $L^{2}\left(\Omega_{0}\right)$. For any $v \in H^{1}\left(\Omega_{0}\right)$ and $v=0$ on $\Gamma_{3}$, it is easy to see that

$$
\begin{aligned}
B\left(u_{\varepsilon}-u_{0}, v\right)= & \int_{\Omega_{\varepsilon}} \nabla\left(u_{\varepsilon}-u_{0}\right) \cdot \nabla v d x \\
& +\int_{\Gamma_{1}^{\varepsilon}} p\left(u_{\varepsilon}-u_{0}\right) v d \sigma_{1} \\
& +\int_{\Gamma_{2}^{\varepsilon}} q\left(u_{\varepsilon}-u_{0}\right) v d \sigma_{2} \\
= & \int_{\Omega_{\varepsilon}} f v d x+\int_{\Gamma_{1}^{\varepsilon}} g v d \sigma_{1}-\int_{\Gamma_{1}^{\varepsilon}} p u_{0} v d \sigma_{1} \\
& -\int_{\Omega_{\varepsilon}} \nabla u_{0} \cdot \nabla v d x+\int_{\Gamma_{2}^{\varepsilon}} h v d \sigma_{2} \\
& -\int_{\Gamma_{2}^{\varepsilon}} q u_{0} v d \sigma_{2} \\
= & -\int_{\Omega_{0}} \nabla u_{0} \cdot \nabla v d x-\int_{\Gamma_{1}} P u_{0} v d x^{\prime} \\
& -\int_{\Gamma_{2}} Q u_{0} v d x^{\prime}+\int_{\Gamma_{1}} P u_{0} v d x^{\prime} \\
& -\int_{\Gamma_{1}^{\varepsilon}} p u_{0} v d \sigma_{1}+\int_{\Gamma_{2}} Q u_{0} v d x^{\prime} \\
& -\int_{\Gamma_{2}^{\varepsilon}} q u_{0} v d \sigma_{2}+\int_{\Omega_{\varepsilon}} f v d x
\end{aligned}
$$

$$
\begin{aligned}
& +\int_{\Gamma_{1}^{\varepsilon}} g v d \sigma_{1}+\int_{\Gamma_{2}^{\varepsilon}} h v d \sigma_{2} \\
& +\int_{\Omega_{0} \backslash \Omega_{\varepsilon}} \nabla u_{0} \cdot \nabla v d x \\
& =-\int_{\Omega_{0} \backslash \Omega_{\varepsilon}} f v d x+\int_{\Omega_{0} \backslash \Omega_{\varepsilon}} \nabla u_{0} \cdot \nabla v d x \\
& +\int_{\Gamma_{1}^{\varepsilon}} g v d \sigma_{1}-\int_{\Gamma_{1}} G v d x^{\prime}+\int_{\Gamma_{2}^{\varepsilon}} h v d \sigma_{2} \\
& -\int_{\Gamma_{2}} H v d x^{\prime}+\int_{\Gamma_{1}} P u_{0} v d x^{\prime} \\
& -\int_{\Gamma_{1}^{\varepsilon}} p u_{0} v d \sigma_{1}+\int_{\Gamma_{2}} Q u_{0} v d x^{\prime} \\
& -\int_{\Gamma_{2}^{\varepsilon}} q u_{0} v d \sigma_{2} .
\end{aligned}
$$

It follows from Proposition 2 that

$$
\left|\int_{\Omega_{0} \backslash \Omega_{\varepsilon}} f v d x\right| \leq C\left(\varepsilon^{\alpha / 2}+\varepsilon^{\beta / 2}\right)\|f\|_{L^{2}\left(\Omega_{0}\right)}\|v\|_{H^{1}\left(\Omega_{0}\right)}
$$

and

$$
\left|\int_{\Omega_{0} \backslash \Omega_{\varepsilon}} \nabla u_{0} \cdot \nabla v d x d x\right| \leq C\left(\varepsilon^{\alpha / 2}+\varepsilon^{\beta / 2}\right)\|v\|_{H^{1}\left(\Omega_{0}\right)},
$$

where we have used the uniform boundedness of $\left\|u_{0}\right\|_{H^{2}\left(\Omega_{0}\right)}$.

Then, according to Propositions 4 and 5 , we conclude that

$$
\begin{aligned}
& \mid \int_{\Omega_{\varepsilon}} \nabla\left(u_{\varepsilon}-u_{0}\right) \cdot \nabla v d x+\int_{\Gamma_{1}^{\varepsilon}} p\left(u_{\varepsilon}-u_{0}\right) v d \sigma_{1} \\
& \quad+\int_{\Gamma_{2}^{\varepsilon}} q\left(u_{\varepsilon}-u_{0}\right) v d \sigma_{2} \mid \leq C\left(\varepsilon^{\alpha / 2}+\varepsilon^{\beta / 2}\right)\|v\|_{H^{1}\left(\Omega_{0}\right)} .
\end{aligned}
$$

Choosing $v=u_{\varepsilon}-u_{0}$ and using condition (4) $(p \geq 0, q \geq 0)$ and Poincaré's inequality, we obtain the desired result. This completes the proof.

\section{Construction of Correctors $u_{1}$ and $u_{2}$}

In order to improve the power of $\varepsilon$, in this section, we shall construct the correctors $u_{1}$ and $u_{2}$.

Firstly, we introduce the harmonic functions $M_{i}\left(x^{\prime}, \xi\right)$, and $x^{\prime}$ is treated as a parameter, as solutions of 


$$
\begin{gathered}
\Delta M_{i}=0 \quad \text { in } \widetilde{D}, \\
\frac{\partial M_{0}}{\partial \nu_{\xi}}=g-\frac{p}{P} G \quad \text { on } \widetilde{\Gamma}, \\
\frac{\partial M_{j}}{\partial \nu_{\xi}}=\frac{-1}{\left[1+\left|\nabla_{\xi^{\prime}} S\left(x^{\prime}, \xi^{\prime}\right)\right|^{2}\right]^{1 / 2}} \frac{\partial S\left(x^{\prime}, \xi^{\prime}\right)}{\partial \xi_{j}} \\
\frac{\partial M_{n}}{\partial \nu_{\xi}}=\frac{1}{\left[1+\left|\nabla \xi^{\prime} S\left(x^{\prime}, \xi^{\prime}\right)\right|^{2}\right]^{1 / 2}-\frac{p}{P} \quad \text { on } \widetilde{\Gamma},} \\
\left|M_{i}\right| \leq C \exp \left(-\lambda \xi_{i}\right) \quad i=0,1, \cdots, n, \lambda>0, \\
M_{i}\left(x^{\prime}, \xi\right) \text { is } 1-\text { periodic in } \xi^{\prime},
\end{gathered}
$$

where

$$
\widetilde{D}=\left\{\left(\xi^{\prime}, \xi_{n}\right): \xi^{\prime} \in(0,1)^{n-1}, S\left(x^{\prime}, \xi^{\prime}\right)<\xi_{n}<+\infty\right\},
$$

and

$$
\widetilde{\Gamma}: \xi_{n}=S\left(x^{\prime}, \xi^{\prime}\right)
$$

This system was first introduced by A. G. Belyaev [13, 14].

To ensure these solutions $M_{i}$ exist, we need to verify the compatibility condition holds true. A simple calculation then gives

$$
\begin{aligned}
& \int_{\partial \widetilde{D}} \frac{\partial M_{0}}{\partial \nu_{\xi}} d \sigma_{\xi} \\
& =\int_{0}^{1} \cdots \int_{0}^{1}\left(g-\frac{p}{P} G\right)\left[1+\left|\nabla_{\xi^{\prime}} S\left(x^{\prime}, \xi^{\prime}\right)\right|^{2}\right]^{1 / 2} d \xi^{\prime} \\
& \quad=0 \\
& \int_{\partial \widetilde{D}} \frac{\partial M_{j}}{\partial \nu_{\xi}} d \sigma_{\xi}=\int_{0}^{1} \cdots \int_{0}^{1}-\frac{\partial S\left(x^{\prime}, \xi^{\prime}\right)}{\partial \xi_{j}} d \xi^{\prime}=0
\end{aligned}
$$

where we have used (5) and

$$
\begin{aligned}
\int_{\partial \widetilde{D}} & \frac{\partial M_{n}}{\partial \nu_{\xi}} d \sigma_{\xi} \\
\quad= & \int_{0}^{1} \cdots \int_{0}^{1}\left(1-\frac{p}{P}\left[1+\left|\nabla_{\xi^{\prime}} S\left(x^{\prime}, \xi^{\prime}\right)\right|^{2}\right]^{1 / 2}\right) d \xi^{\prime} \\
& =0 .
\end{aligned}
$$

Therefore, these harmonic functions $M_{i}$ exist.

Then we define $u_{1}$ as follows:

$$
u_{1}(x, \xi)=M_{0}\left(x^{\prime}, \xi\right)+\sum_{k=1}^{n} \frac{\partial u_{0}}{\partial x_{k}}(x) M_{k}\left(x^{\prime}, \xi\right)
$$

Using the same technique, we construct corrector $u_{2}$ as follows.

Assume harmonic functions $N_{i}\left(x^{\prime}, \eta\right), x^{\prime}$ is treated as a parameter, as solutions of

$$
\begin{gathered}
\Delta N_{i}=0 \quad \text { in } \widehat{D}, \\
\frac{\partial N_{0}}{\partial \nu_{\eta}}=h-\frac{q}{Q} H \quad \text { on } \widehat{\Gamma}, \\
\frac{\partial N_{j}}{\partial \nu_{\eta}}=\frac{-1}{\left[1+\left|\nabla_{\eta^{\prime}} T\left(x^{\prime}, \eta^{\prime}\right)\right|^{2}\right]^{1 / 2}} \frac{\partial T\left(x^{\prime}, \eta^{\prime}\right)}{\partial \eta_{j}} \\
\frac{\partial N_{n}}{\partial \nu_{\eta}}=\frac{-1}{\left[1+\left|\nabla_{\eta^{\prime}} T\left(x^{\prime}, \eta^{\prime}\right)\right|^{2}\right]^{1 / 2}}+\frac{q}{Q} \quad \text { on } \widehat{\Gamma}, \\
\left|N_{i}\right| \leq C \exp \left(-\theta \eta_{i}\right) \quad i=0,1, \ldots, n, \theta>0, \\
N_{i}\left(x^{\prime}, \eta^{\prime}\right) \text { is } 1-\text { periodic in } \eta^{\prime},
\end{gathered}
$$

where

$$
\begin{aligned}
\widehat{D} & =\left\{\left(\eta^{\prime}, \eta_{n}\right): \eta^{\prime} \in(0,1)^{n-1},-\infty<\eta_{n}<1\right. \\
& \left.-T\left(x^{\prime}, \eta^{\prime}\right)\right\},
\end{aligned}
$$

$v_{\eta}=\left(v_{\eta}^{1}, v_{\eta}^{2} \cdots, v_{\eta}^{n}\right)$ is the outward normal,

and the hypersurface

$$
\widehat{\Gamma}: \eta_{n}=T\left(x^{\prime}, \eta^{\prime}\right) .
$$

It is easy to verify the compatibility condition holds true. We define $u_{2}$ as follows:

$$
u_{2}(x, \eta)=N_{0}\left(x^{\prime}, \eta\right)+\sum_{k=1}^{n} \frac{\partial u_{0}}{\partial x_{k}}(x) N_{k}\left(x^{\prime}, \eta\right) \text {. }
$$

\section{Error Estimate up to $O\left(\varepsilon^{\alpha}+\varepsilon^{\beta}\right)$}

In this section, we will prove the error estimate up to the order of $O\left(\varepsilon^{\alpha}+\varepsilon^{\beta}\right)$. The main technique is using the correctors $u_{1}$ and $u_{2}$. Our main result is the following theorem.

Theorem 8 (let $f \in L^{2}\left(\Omega_{\varepsilon}\right)$ ). Assume that $u_{\varepsilon}$ is a solution of problem (1)-(3). Suppose that $p\left(x^{\prime}, \xi^{\prime}\right), g\left(x^{\prime}, \xi^{\prime}\right), q\left(x^{\prime}, \eta^{\prime}\right)$, $h\left(x^{\prime}, \eta^{\prime}\right), S\left(x^{\prime}, \xi^{\prime}\right)$, and $T\left(x^{\prime}, \eta^{\prime}\right)$ satisfy (4)-(6). Then there exists a constant $C$ which does not depend on $\varepsilon$, such that

$$
\left\|u_{\varepsilon}-u_{0}-\varepsilon^{\alpha} u_{1}-\varepsilon^{\beta} u_{2}\right\|_{H^{1}\left(\Omega_{\varepsilon}\right)} \leq C\left(\varepsilon^{\alpha}+\varepsilon^{\beta}\right),
$$

where correctors $u_{1}$ and $u_{2}$ are defined in (38) and (42), respectively.

Proof. Analogously to Section 3, for any $v \in H^{1}\left(\Omega_{0}\right)$ and $v=$ 0 on $\Gamma_{3}$, we consider

$$
\begin{aligned}
B\left(u_{\varepsilon}-u_{0}-\varepsilon^{\alpha} u_{1}-\varepsilon^{\beta} u_{2}, v\right) \\
=B\left(u_{\varepsilon}-u_{0}, v\right)-\int_{\Omega_{\varepsilon}} \nabla_{\xi} u_{1} \cdot \nabla v d x \\
\quad-\int_{\Omega_{\varepsilon}} \nabla_{\eta} u_{2} \cdot \nabla v d x+O\left(\varepsilon^{\alpha}+\varepsilon^{\beta}\right)\|v\|_{H^{1}\left(\Omega_{0}\right)} .
\end{aligned}
$$

Firstly, let us estimate the term $B\left(u_{\varepsilon}-u_{0}, v\right)$. Clearly, 


$$
\begin{aligned}
B\left(u_{\varepsilon}-u_{0}, v\right)= & \int_{\Omega_{\varepsilon}} \nabla\left(u_{\varepsilon}-u_{0}\right) \cdot \nabla v d x \\
& +\int_{\Gamma_{1}^{\varepsilon}} p\left(u_{\varepsilon}-u_{0}\right) v d \sigma_{1} \\
& +\int_{\Gamma_{2}^{\varepsilon}} q\left(u_{\varepsilon}-u_{0}\right) v d \sigma_{2} \\
= & \int_{\Gamma_{1}^{\varepsilon}}\left(-p u_{0}-\frac{\partial u_{0}}{\partial \nu_{\varepsilon}}+g\right) v d \sigma_{1} \\
& +\int_{\Gamma_{2}^{\varepsilon}}\left(-q u_{0}-\frac{\partial u_{0}}{\partial \nu_{\varepsilon}}+h\right) v d \sigma_{2}
\end{aligned}
$$

It follows from (7) that

$$
\begin{aligned}
& \frac{\partial u_{0}}{\partial v_{0}}+P\left(x^{\prime}\right) u_{0}=G\left(x^{\prime}\right) \text { on } \Gamma_{1}, \\
& \frac{\partial u_{0}}{\partial v_{0}}+Q\left(x^{\prime}\right) u_{0}=H\left(x^{\prime}\right) \text { on } \Gamma_{2} .
\end{aligned}
$$

Hence

$$
\begin{gathered}
\left|p u_{0}+\frac{p}{P}\left(\frac{\partial u_{0}}{\partial \nu_{0}}-G\right)\right|_{\Gamma_{1}^{\varepsilon}} \leq C \varepsilon^{\alpha}, \\
\left|q u_{0}+\frac{q}{Q}\left(\frac{\partial u_{0}}{\partial \nu_{0}}-H\right)\right|_{\Gamma_{2}^{\varepsilon}} \leq C \varepsilon^{\beta} .
\end{gathered}
$$

This gives

$$
\begin{aligned}
B\left(u_{\varepsilon}-u_{0}, v\right)= & \int_{\Gamma_{1}^{\varepsilon}}\left(g-\frac{p}{P} G\right) v d \sigma_{1} \\
& +\int_{\Gamma_{1}^{\varepsilon}}\left(\frac{p}{P} \frac{\partial u_{0}}{\partial \nu_{0}}-\frac{\partial u_{0}}{\partial \nu_{\varepsilon}}\right) v d \sigma_{1}
\end{aligned}
$$

$$
+O\left(\varepsilon^{\alpha}\right)\|v\|_{H^{1}\left(\Omega_{0}\right)}
$$$$
+\int_{\Gamma_{2}^{\varepsilon}}\left(h-\frac{q}{Q} H\right) v d \sigma_{2}
$$$$
+\int_{\Gamma_{2}^{\varepsilon}}\left(\frac{q}{Q} \frac{\partial u_{0}}{\partial \nu_{0}}-\frac{\partial u_{0}}{\partial \nu_{\varepsilon}}\right) v d \sigma_{2}
$$$$
+O\left(\varepsilon^{\beta}\right)\|v\|_{H^{1}\left(\Omega_{0}\right)} \text {. }
$$

In view of (44), we obtain

$$
\begin{aligned}
& B\left(u_{\varepsilon}-u_{0}-\varepsilon^{\alpha} u_{1}-\varepsilon^{\beta} u_{2}, v\right) \\
& =\int_{\Gamma_{1}^{\varepsilon}}\left(g-\frac{p}{P} G\right) v d \sigma_{1}+\int_{\Gamma_{1}^{\varepsilon}}\left(\frac{p}{P} \frac{\partial u_{0}}{\partial \nu_{0}}-\frac{\partial u_{0}}{\partial \nu_{\varepsilon}}\right) v d \sigma_{1} \\
& \quad-\int_{\Omega_{\varepsilon}} \nabla \xi u_{1} \cdot \nabla v d x+O\left(\varepsilon^{\alpha}\right)\|v\|_{H^{1}\left(\Omega_{0}\right)} \\
& \quad+\int_{\Gamma_{2}^{\varepsilon}}\left(h-\frac{q}{Q} H\right) v d \sigma_{2} \\
& \quad+\int_{\Gamma_{2}^{\varepsilon}}\left(\frac{q}{Q} \frac{\partial u_{0}}{\partial v_{0}}-\frac{\partial u_{0}}{\partial v_{\varepsilon}}\right) v d \sigma_{2}-\int_{\Omega_{\varepsilon}} \nabla_{\eta} u_{2} \cdot \nabla v d x \\
& \quad+O\left(\varepsilon^{\beta}\right)\|v\|_{H^{1}\left(\Omega_{0}\right)} .
\end{aligned}
$$

We shall next deal with the term $\int_{\Omega_{\varepsilon}} \nabla_{\xi} u_{1} \cdot \nabla v d x$. Using the change of variables, we find that

$$
\int_{\Omega_{\varepsilon}} \nabla \xi u_{1} \cdot \nabla v d x=\varepsilon^{(n-1) \alpha} \sum_{i} \int_{\widetilde{\Omega}_{i}} \nabla_{\xi} u_{1} \cdot \nabla_{\xi} v d \xi
$$

where

$$
\widetilde{\Omega_{i}}=\left\{\left(\xi^{\prime}, \xi_{n}\right) \mid \xi^{\prime} \in\left(0, \frac{1}{\varepsilon^{\alpha}}\right)^{n-1}, S<\xi_{n}<\frac{1}{\varepsilon^{\alpha}}-\varepsilon^{\beta-\alpha} T\right\} \cap\left\{\xi^{\prime} \in\left(\frac{A_{i}}{\varepsilon^{\alpha}}, \frac{A_{i+1}}{\varepsilon^{\alpha}}\right)^{n-1}\right\},
$$

where $A_{i+1}-A_{i}=\varepsilon^{\alpha}, i=0,1,2 \cdots$, and we assume that $A_{0}=0$. we get

In view of integration by parts and the fact that $\Delta_{\xi} u_{1}=0$,

$$
\int_{\Omega_{\varepsilon}} \nabla_{\xi} u_{1} \cdot \nabla v d x=\varepsilon^{(n-1) \alpha} \sum_{i} \int_{\widetilde{\Gamma}_{i}} \frac{\partial u_{1}}{\partial \nu_{\xi}} v d \sigma_{\xi}
$$

where

$$
\widetilde{\Gamma_{i}}:\left\{\xi_{n}=S\left(x^{\prime}, \xi^{\prime}\right), \xi_{i} \in\left(\frac{A_{i}}{\varepsilon^{\alpha}}, \frac{A_{i+1}}{\varepsilon^{\alpha}}\right)^{n-1}\right\} .
$$

$$
\begin{aligned}
& \text { It follows from (38) that } \\
& \begin{array}{l}
\int_{\Omega_{\varepsilon}} \nabla_{\xi} u_{1} \cdot \nabla v d x=\varepsilon^{(n-1) \alpha} \sum_{i}\left(\int_{\widetilde{\Gamma}_{i}} \frac{\partial M_{0}}{\partial \nu_{\xi}} v d \sigma_{\xi}\right. \\
\left.\quad+\sum_{j=1}^{n-1} \int_{\widetilde{\Gamma_{i}}} \frac{\partial M_{j}}{\partial \nu_{\xi}} \frac{\partial u_{0}}{\partial x_{j}} v d \sigma_{\xi}+\int_{\widetilde{\Gamma_{i}}} \frac{\partial M_{n}}{\partial \nu_{\xi}} \frac{\partial u_{0}}{\partial x_{n}} v d \sigma_{\xi}\right) \\
\quad \doteq A_{1}+A_{2}+A_{3},
\end{array}
\end{aligned}
$$




$$
\begin{aligned}
A_{1} & =\varepsilon^{(n-1) \alpha} \sum_{i} \int_{\widetilde{\Gamma_{i}}} \frac{\partial M_{0}}{\partial v_{\xi}} v d \sigma_{\xi}=\varepsilon^{(n-1) \alpha} \sum_{i} \int_{\widetilde{\Gamma}_{i}}\left(g-\frac{p}{P} G\right) v d \sigma_{\xi} \\
& =\varepsilon^{(n-1) \alpha} \sum_{i} \int_{A_{i} / \varepsilon^{\alpha}}^{A_{i+1} / \varepsilon^{\alpha}} \cdots \int_{A_{i} / \varepsilon^{\alpha}}^{A_{i+1} / \varepsilon^{\alpha}}\left(g-\frac{p}{P} G\right) v\left[1+\left|\nabla_{\xi^{\prime}} S\left(x^{\prime}, \xi^{\prime}\right)\right|^{2}\right]^{1 / 2} d \xi^{\prime}, \\
A_{2} & =\varepsilon^{(n-1) \alpha} \sum_{j=1}^{n-1} \sum_{i} \int_{\widetilde{\Gamma_{i}}} \frac{\partial M_{j}}{\partial \nu_{\xi}} \frac{\partial u_{0}}{\partial x_{j}} v d \sigma_{\xi}=\varepsilon^{(n-1) \alpha} \sum_{j=1}^{n-1} \sum_{i} \int_{\widetilde{\Gamma}_{i}} \frac{-1}{\left[1+\left|\nabla_{\xi^{\prime}} S\left(x^{\prime}, \xi^{\prime}\right)\right|^{2}\right]^{1 / 2}} \frac{\partial S\left(x^{\prime}, \xi^{\prime}\right)}{\partial \xi_{j}} \frac{\partial u_{0}}{\partial x_{j}} v d \sigma_{\xi} \\
& =\varepsilon^{(n-1) \alpha} \sum_{j=1}^{n-1} \sum_{i} \int_{A_{i} / \varepsilon^{\alpha}}^{A_{i+1} / \varepsilon^{\alpha}} \cdots \int_{A_{i} / \varepsilon^{\alpha}}^{A_{i+1} / \varepsilon^{\alpha}}-\frac{\partial S\left(x^{\prime}, \xi^{\prime}\right)}{\partial \xi_{j}} \frac{\partial u_{0}}{\partial x_{j}} v d \xi^{\prime}, \text { and } \\
A_{3} & =\varepsilon^{(n-1) \alpha} \sum_{i} \int_{\widetilde{\Gamma}_{i}} \frac{\partial M_{n}}{\partial \nu_{\xi}} \frac{\partial u_{0}}{\partial x_{n}} v d \sigma_{\xi}=\varepsilon^{(n-1) \alpha} \sum_{i} \int_{\widetilde{\Gamma}_{i}}\left(\frac{p}{\left[1+\left|\nabla \xi^{\prime} S\left(x^{\prime}, \xi^{\prime}\right)\right|^{2}\right]^{1 / 2}}-\frac{p}{P}\right) \frac{\partial u_{0}}{\partial x_{n}} v d \sigma_{\xi} \\
& =\varepsilon^{(n-1) \alpha} \sum_{i} \int_{A_{i} / \varepsilon^{\alpha}}^{A_{i+1} / \varepsilon^{\alpha}} \cdots \int_{A_{i} / \varepsilon^{\alpha}}^{A_{i+1} / \varepsilon^{\alpha}}\left(1-\frac{p}{P}\left[1+\left|\nabla_{\xi^{\prime}} S\left(x^{\prime}, \xi^{\prime}\right)\right|^{2}\right]^{1 / 2}\right) \frac{\partial u_{0}}{\partial x_{n}} v d \xi^{\prime} .
\end{aligned}
$$

Also, note that

$$
\begin{gathered}
\int_{\Gamma_{1}^{\varepsilon}}\left(g-\frac{p}{P} G\right) v d \sigma_{1}=\int_{\Gamma_{1}}\left(g-\frac{p}{P} G\right) v[1 \\
\left.+\left|\nabla_{\xi^{\prime}} S\left(x^{\prime}, \xi^{\prime}\right)\right|^{2}\right]^{1 / 2} d x^{\prime}+O\left(\varepsilon^{\alpha}\right)\|v\|_{H^{1}\left(\Omega_{0}\right)} \\
=\varepsilon^{(n-1) \alpha} \sum_{i} \int_{A_{i} / \varepsilon^{\alpha}}^{A_{i+1} / \varepsilon^{\alpha}} \cdots \int_{A_{i} / \varepsilon^{\alpha}}^{A_{i+1} / \varepsilon^{\alpha}}\left(g-\frac{p}{P} G\right) \\
\cdot v\left[1+\left|\nabla_{\xi^{\prime}} S\left(x^{\prime}, \xi^{\prime}\right)\right|^{2}\right]^{1 / 2} d \xi^{\prime}+O\left(\varepsilon^{\alpha}\right)
\end{gathered}
$$

$\cdot\|v\|_{H^{1}\left(\Omega_{0}\right)}$, where we have used the change of variables $x^{\prime}=\varepsilon^{\alpha} \xi^{\prime}$. Hence

$$
\int_{\Gamma_{1}^{\varepsilon}}\left(g-\frac{p}{P} G\right) v d \sigma_{1}-A_{1}=O\left(\varepsilon^{\alpha}\right)\|v\|_{H^{1}\left(\Omega_{0}\right)} .
$$

Next, we shall evaluate the term $\int_{\Gamma_{1}^{\varepsilon}}\left((p / P)\left(\partial u_{0} / \partial v_{0}\right)-\right.$ $\left.\partial u_{0} / \partial v_{\varepsilon}\right) v d \sigma_{1}$.

Also, note that since $v_{0}=(0, \ldots, 0,-1)$ and $v_{\varepsilon}=\left(\nabla_{\xi^{\prime}} S\right.$, $-1)\left(1 /\left[1+\left|\nabla_{\xi^{\prime}} S\left(x^{\prime}, \xi^{\prime}\right)\right|^{2}\right]^{1 / 2}\right)+O\left(\varepsilon^{\alpha}\right)$,

$$
\begin{aligned}
\int_{\Gamma_{1}^{\varepsilon}}\left(\frac{p}{P} \frac{\partial u_{0}}{\partial v_{0}}-\frac{\partial u_{0}}{\partial v_{\varepsilon}}\right) v d \sigma_{1}= & \int_{\Gamma_{1}^{\varepsilon}}\left(\frac{p}{P} \nabla u_{0} \cdot v_{0}-\nabla u_{0} \cdot v_{\varepsilon}\right) v d \sigma_{1} \\
= & \int_{\Gamma_{1}^{\varepsilon}} \frac{\partial u_{0}}{\partial x_{n}}\left(\frac{1}{\left[1+\left|\nabla_{\xi^{\prime}} S\left(x^{\prime}, \xi^{\prime}\right)\right|^{2}\right]^{1 / 2}}-\frac{p}{P}\right) v d \sigma_{1} \\
& -\sum_{j=1}^{n-1} \int_{\Gamma_{1}^{\varepsilon}} \frac{\partial u_{0}}{\partial x_{j}} \frac{1}{\left[1+\left|\nabla_{\xi^{\prime}} S\left(x^{\prime}, \xi^{\prime}\right)\right|^{2}\right]^{1 / 2}} \frac{\partial S}{\partial \xi_{j}} v d \sigma_{1}+O\left(\varepsilon^{\alpha}\right)\|v\|_{H^{1}\left(\Omega_{0}\right)} \\
= & \int_{\Gamma_{1}} \frac{\partial u_{0}}{\partial x_{n}}\left(1-\frac{p}{P}\left[1+\left|\nabla \xi^{\prime} S\left(x^{\prime}, \xi^{\prime}\right)\right|^{2}\right]^{1 / 2}\right) v d x^{\prime}-\sum_{j=1}^{n-1} \int_{\Gamma_{1}} \frac{\partial u_{0}}{\partial x_{j}} \frac{\partial S}{\partial \xi_{j}} v d x^{\prime}+O\left(\varepsilon^{\alpha}\right)\|v\|_{H^{1}\left(\Omega_{0}\right)} \\
= & \varepsilon^{(n-1) \alpha} \sum_{i} \int_{A_{i} / \varepsilon^{\alpha}}^{A_{i+1} / \varepsilon^{\alpha}} \ldots \int_{A_{i} / \varepsilon^{\alpha}}^{A_{i+1} / \varepsilon^{\alpha}} \frac{\partial u_{0}}{\partial x_{n}}\left(1-\frac{p}{P}\left[1+\left|\nabla \xi^{\prime} S\left(x^{\prime}, \xi^{\prime}\right)\right|^{2}\right]^{1 / 2}\right) v d \xi^{\prime} \\
& -\varepsilon^{(n-1) \alpha} \sum_{j=1}^{n-1} \sum_{i} \int_{A_{i} / \varepsilon^{\alpha}}^{A_{i+1} / \varepsilon^{\alpha}} \cdots \int_{A_{i} / \varepsilon^{\alpha}}^{A_{i+1} / \varepsilon^{\alpha}} \frac{\partial u_{0}}{\partial x_{j}} \frac{\partial S}{\partial \xi_{j}} v d \xi^{\prime}+O\left(\varepsilon^{\alpha}\right)\|v\|_{H^{1}\left(\Omega_{0}\right)} .
\end{aligned}
$$


By the definition of $A_{2}$ and $A_{3}$ in (54), this implies that

$$
\begin{aligned}
& \int_{\Gamma_{1}^{\varepsilon}}\left(\frac{p}{P} \frac{\partial u_{0}}{\partial \nu_{0}}-\frac{\partial u_{0}}{\partial \nu_{\varepsilon}}\right) v d \sigma_{1}-A_{2}-A_{3} \\
& =O\left(\varepsilon^{\alpha}\right)\|v\|_{H^{1}\left(\Omega_{0}\right)} .
\end{aligned}
$$

In a similar way, we obtain that

$$
\int_{\Omega_{\varepsilon}} \nabla_{\eta} u_{2} \cdot \nabla v d x \doteq B_{1}+B_{2}+B_{3},
$$

where

$$
\begin{aligned}
& B_{1}=\varepsilon^{(n-1) \alpha} \sum_{i} \int_{B_{i} / \varepsilon^{\beta}}^{B_{i+1} / \varepsilon^{\beta}} \cdots \int_{B_{i} / \varepsilon^{\beta}}^{B_{i+1} / \varepsilon^{\beta}}\left(h-\frac{q}{Q} H\right) v\left[1+\left|\nabla_{\eta^{\prime}} T\left(x^{\prime}, \eta^{\prime}\right)\right|^{2}\right]^{1 / 2} d \eta^{\prime}, \\
& B_{2}=\varepsilon^{(n-1) \alpha} \sum_{j=1}^{n-1} \sum_{i} \int_{B_{i} / \varepsilon^{\beta}}^{B_{i+1} / \varepsilon^{\beta}} \cdots \int_{B_{i} / \varepsilon^{\beta}}^{B_{i+1} / \varepsilon^{\beta}}-\frac{\partial T\left(x^{\prime}, \eta^{\prime}\right)}{\partial \eta_{j}} \frac{\partial u_{0}}{\partial x_{j}} v d \eta^{\prime}, \\
& B_{3}=\varepsilon^{(n-1) \alpha} \sum_{i} \int_{B_{i} / \varepsilon^{\beta}}^{B_{i+1} / \varepsilon^{\beta}} \cdots \int_{B_{i} / \varepsilon^{\beta}}^{B_{i+1} / \varepsilon^{\beta}}\left(-1+\frac{q}{Q}\left[1+\left|\nabla_{\eta^{\prime}} T\left(x^{\prime}, \eta^{\prime}\right)\right|^{2}\right]^{1 / 2}\right) \frac{\partial u_{0}}{\partial x_{n}} v d \eta^{\prime} .
\end{aligned}
$$

Using the same technique, we also have

$$
\begin{aligned}
& \int_{\Gamma_{2}^{\varepsilon}}\left(h-\frac{q}{Q} H\right) v d \sigma_{2}-B_{1}=O\left(\varepsilon^{\beta}\right)\|v\|_{H^{1}\left(\Omega_{0}\right)} \text { and } \\
& \int_{\Gamma_{2}^{\varepsilon}}\left(\frac{q}{Q} \frac{\partial u_{0}}{\partial \nu_{0}}-\frac{\partial u_{0}}{\partial \nu_{\varepsilon}}\right) v d \sigma_{2}-B_{2}-B_{3} \\
& =O\left(\varepsilon^{\beta}\right)\|v\|_{H^{1}\left(\Omega_{0}\right)} .
\end{aligned}
$$

Combining all these terms and choosing $v=u_{\varepsilon}-u_{0}-$ $\varepsilon^{\alpha}\left(u_{1}-\left.u_{1}\right|_{x_{1}=1}\right)-\varepsilon^{\beta}\left(u_{2}-\left.u_{2}\right|_{x_{1}=1}\right)$, we conclude that

$$
\left\|u_{\varepsilon}-u_{0}-\varepsilon^{\alpha} u_{1}-\varepsilon^{\beta} u_{2}\right\|_{H^{1}\left(\Omega_{\varepsilon}\right)} \leq C\left(\varepsilon^{\alpha}+\varepsilon^{\beta}\right),
$$

where we have used Poincaré's inequality and the fact

$$
\begin{aligned}
& \left.\varepsilon^{\alpha} u_{1}\right|_{x_{i}=1}+\left.\varepsilon^{\beta} u_{2}\right|_{x_{i}=1} \\
& \quad \leq C\left(\varepsilon^{\alpha}+\varepsilon^{\beta}\right)\left[\exp \left(-\frac{\lambda}{\varepsilon^{\alpha}}\right)+\exp \left(-\frac{\theta}{\varepsilon^{\beta}}\right)\right],
\end{aligned}
$$

where $i=1,2 \ldots, n-1$.

This completes the proof.

\section{Error Estimate up to $O\left(\varepsilon^{3 \alpha / 2}+\varepsilon^{3 \beta / 2}\right)$}

In this section, we will improve the error estimate up to the order of $O\left(\varepsilon^{3 \alpha / 2}+\varepsilon^{3 \beta / 2}\right)$. The main technique is using the correctors and second-order Taylor's expansion. Our main result is the following theorem.

Theorem 9 (let $f \in L^{2}\left(\Omega_{\varepsilon}\right)$ ). Assume that $u_{\varepsilon}$ is a solution of problem (1)-(3). Suppose that $p\left(x^{\prime}, \xi^{\prime}\right), g\left(x^{\prime}, \xi^{\prime}\right), q\left(x^{\prime}, \eta^{\prime}\right)$, $h\left(x^{\prime}, \eta^{\prime}\right), S\left(x^{\prime}, \xi^{\prime}\right)$, and $T\left(x^{\prime}, \eta^{\prime}\right)$ satisfy (4)-(6). Then there exists a constant $C$ that does not depend on $\varepsilon$, such that

$$
\begin{aligned}
& \left\|u_{\varepsilon}-u_{0}-\varepsilon^{\alpha} u_{1}-\varepsilon^{\beta} u_{2}+\varepsilon^{\alpha} \phi_{1}+\varepsilon^{\beta} \phi_{2}\right\|_{H^{1}\left(\Omega_{\varepsilon}\right)} \\
& \leq C\left(\varepsilon^{3 \alpha / 2}+\varepsilon^{3 \beta / 2}\right),
\end{aligned}
$$

where correctors $\phi_{1}$ and $\phi_{2}$ are given by (88) and (93).

Proof. Following the same line of research, we consider

$$
\begin{aligned}
B\left(u_{\varepsilon}-u_{0}, v\right)= & \int_{\Gamma_{1}^{\varepsilon}}\left(-p u_{0}-\frac{\partial u_{0}}{\partial \nu_{\varepsilon}}+g\right) v d \sigma_{1} \\
& +\int_{\Gamma_{2}^{\varepsilon}}\left(-q u_{0}-\frac{\partial u_{0}}{\partial \nu_{\varepsilon}}+h\right) v d \sigma_{2} .
\end{aligned}
$$

This, together with (7) and the second-order Taylor's expansion, gives

$$
\begin{aligned}
& \left(\frac{\partial u_{0}}{\partial \nu_{0}}+P\left(x^{\prime}\right) u_{0}-G\left(x^{\prime}\right)\right)_{\Gamma_{1}^{\varepsilon}} \\
& =\varepsilon^{\alpha} S\left(x^{\prime}, \xi^{\prime}\right) \frac{\partial}{\partial x_{n}}\left(\frac{\partial u_{0}}{\partial \nu_{0}}+P\left(x^{\prime}\right) u_{0}-G\left(x^{\prime}\right)\right)_{\Gamma_{1}} \\
& \quad+O\left(\varepsilon^{2 \alpha}\right), \text { and } \\
& \left(\frac{\partial u_{0}}{\partial v_{0}}+Q\left(x^{\prime}\right) u_{0}-H\left(x^{\prime}\right)\right)_{\Gamma_{2}^{\varepsilon}} \\
& =-\varepsilon^{\beta} T\left(x^{\prime}, \eta^{\prime}\right) \frac{\partial}{\partial x_{n}}\left(\frac{\partial u_{0}}{\partial v_{0}}+Q\left(x^{\prime}\right) u_{0}-H\left(x^{\prime}\right)\right)_{\Gamma_{2}} \\
& \quad+O\left(\varepsilon^{2 \beta}\right) .
\end{aligned}
$$

It follows that 


$$
\begin{aligned}
& B\left(u_{\varepsilon}-u_{0}, v\right) \\
& =\int_{\Gamma_{1}^{\varepsilon}}\left(g-\frac{p}{P} G\right) v d \sigma_{1}+\int_{\Gamma_{1}^{\varepsilon}}\left(\frac{p}{P} \frac{\partial u_{0}}{\partial v_{0}}-\frac{\partial u_{0}}{\partial v_{\varepsilon}}\right) v d \sigma_{1} \\
& \quad-\int_{\Gamma_{1}^{\varepsilon}} \varepsilon^{\alpha} \frac{p}{P} S\left(x^{\prime}, \xi^{\prime}\right) \frac{\partial}{\partial x_{n}}\left(\frac{\partial u_{0}}{\partial v_{0}}+P\left(x^{\prime}\right) u_{0}\right) v d \sigma_{1} \\
& \quad+O\left(\varepsilon^{2 \alpha}\right)\|v\|_{H^{1}\left(\Omega_{0}\right)}+\int_{\Gamma_{2}^{e}}\left(h-\frac{q}{Q} H\right) v d \sigma_{2} \\
& \quad+\int_{\Gamma_{2}^{\varepsilon}}\left(\frac{q}{Q} \frac{\partial u_{0}}{\partial v_{0}}-\frac{\partial u_{0}}{\partial v_{\varepsilon}}\right) v d \sigma_{2} \\
& \quad+\int_{\Gamma_{2}^{\varepsilon}} \varepsilon^{\beta} \frac{q}{Q} T\left(x^{\prime}, \eta^{\prime}\right) \frac{\partial}{\partial x_{n}}\left(\frac{\partial u_{0}}{\partial v_{0}}+Q\left(x^{\prime}\right) u_{0}\right) v d \sigma_{2} \\
& \quad+O\left(\varepsilon^{2 \beta}\right)\|v\|_{H^{1}\left(\Omega_{0}\right) .}
\end{aligned}
$$

Note that

$$
\begin{aligned}
& B\left(-\varepsilon^{\alpha} u_{1}-\varepsilon^{\beta} u_{2}, v\right)=\int_{\Omega_{\varepsilon}} \varepsilon^{\alpha} \Delta u_{1} v d x \\
& -\int_{\Gamma_{1}^{\varepsilon}} \varepsilon^{\alpha} \frac{\partial u_{1}}{\partial \nu_{\varepsilon}} v d \sigma_{1} \\
& -\int_{\Gamma_{1}^{\varepsilon}} \varepsilon^{\alpha} p u_{1} v d \sigma_{1} \\
& +\int_{\Omega_{\varepsilon}} \varepsilon^{\beta} \Delta u_{2} v d x \\
& -\int_{\Gamma_{2}^{\varepsilon}} \varepsilon^{\beta} \frac{\partial u_{2}}{\partial \nu_{\varepsilon}} v d \sigma_{2} \\
& -\int_{\Gamma_{2}^{\varepsilon}} \varepsilon^{\beta} q u_{2} v d \sigma_{2} \\
& =\int_{\Omega_{\varepsilon}} \varepsilon^{\alpha} \triangle_{x} u_{1} v d x \\
& +\int_{\Omega_{\varepsilon}} 2 \nabla_{x} \cdot \nabla_{\xi} u_{1} v d x \\
& -\int_{\Gamma_{1}^{\varepsilon}} \varepsilon^{\alpha} \frac{\partial u_{1}}{\partial \nu_{\varepsilon}} v d \sigma_{1} \\
& +\int_{\Omega_{\varepsilon}} \varepsilon^{\beta} \triangle_{x} u_{2} v d x \\
& +\int_{\Omega_{\varepsilon}} 2 \nabla_{x} \cdot \nabla_{\eta} u_{2} v d x \\
& -\int_{\Gamma_{1}^{\varepsilon}} \varepsilon^{\alpha} p u_{1} v d \sigma_{1} \\
& -\int_{\Gamma_{2}^{\varepsilon}} \varepsilon^{\beta} \frac{\partial u_{2}}{\partial \nu_{\varepsilon}} v d \sigma_{2} \\
& -\int_{\Gamma_{2}^{\varepsilon}} \varepsilon^{\beta} q u_{2} v d \sigma_{2},
\end{aligned}
$$

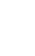

Thus we obtain

$$
\begin{aligned}
& B\left(u_{\varepsilon}-u_{0}-\varepsilon^{\alpha} u_{1}-\varepsilon^{\beta} u_{2}, v\right) \\
& =\int_{\Gamma_{1}^{e}}\left(g-\frac{p}{P} G\right) v d \sigma_{1} \\
& +\int_{\Gamma_{1}^{\varepsilon}}\left(\frac{p}{P} \frac{\partial u_{0}}{\partial v_{0}}-\frac{\partial u_{0}}{\partial v_{\varepsilon}}\right) v d \sigma_{1} \\
& +\int_{\Omega^{\varepsilon}} 2 \nabla_{x} \nabla_{\xi} u_{1} v d x \\
& -\left.\int_{\Gamma_{1}^{\varepsilon}} \varepsilon^{\alpha} \frac{p}{P} S\left(x^{\prime}, \xi^{\prime}\right) \frac{\partial}{\partial x_{n}}\left(\frac{\partial u_{0}}{\partial v_{0}}+P\left(x^{\prime}\right) u_{0}\right)\right|_{\Gamma_{1}} v d \sigma_{1} \\
& +O\left(\varepsilon^{2 \alpha}\right)\|v\|_{H^{1}\left(\Omega_{0}\right)}, \\
& +\int_{\Omega_{\varepsilon}} \varepsilon^{\alpha} \Delta_{x} u_{1} v d x \\
& -\int_{\Gamma_{1}^{\varepsilon}} \varepsilon^{\alpha} p u_{1} v d \sigma_{1} \\
& -\int_{\Gamma_{1}^{\varepsilon}} \varepsilon^{\alpha} \frac{\partial u_{1}}{\partial v_{\varepsilon}} v d \sigma_{1} \\
& +\int_{\Gamma_{2}^{\varepsilon}}\left(h-\frac{q}{Q} H\right) v d \sigma_{2} \\
& +\int_{\Gamma_{2}^{\varepsilon}}\left(\frac{q}{Q} \frac{\partial u_{0}}{\partial \nu_{0}}-\frac{\partial u_{0}}{\partial \nu_{\varepsilon}}\right) v d \sigma_{2} \\
& +\int_{\Omega^{\varepsilon}} 2 \nabla_{x} \nabla_{\eta} u_{2} v d x \\
& +\left.\int_{\Gamma_{2}^{\varepsilon}} \varepsilon^{\beta} \frac{q}{Q} T\left(x^{\prime}, \eta^{\prime}\right) \frac{\partial}{\partial x_{n}}\left(\frac{\partial u_{0}}{\partial \nu_{0}}+Q\left(x^{\prime}\right) u_{0}\right)\right|_{\Gamma_{2}} v d \sigma_{2} \\
& +O\left(\varepsilon^{2 \beta}\right)\|v\|_{H^{1}\left(\Omega_{0}\right)}, \\
& +\int_{\Omega_{\varepsilon}} \varepsilon^{\beta} \triangle_{x} u_{2} v d x \\
& -\int_{\Gamma_{2}^{\varepsilon}} \varepsilon^{\beta} q u_{2} v d \sigma_{2} \\
& -\int_{\Gamma_{2}^{\varepsilon}} \varepsilon^{\beta} \frac{\partial u_{2}}{\partial v_{\varepsilon}} v d \sigma_{2} .
\end{aligned}
$$

Note that on $\Gamma_{1}^{\varepsilon}$

$$
\begin{aligned}
v_{0}= & (0, \cdots, 0,-1), \\
v_{\varepsilon}= & \left(\nabla_{\xi^{\prime}} S,-1\right) \frac{1}{\left[1+\left|\nabla_{\xi^{\prime}} S\right|^{2}\right]^{1 / 2}} \\
& +\varepsilon^{\alpha}\left(\nabla_{x^{\prime}} S, \nabla_{x^{\prime}} S \cdot \nabla_{\xi^{\prime}} S\right) \frac{1}{\left[1+\left|\nabla_{\xi^{\prime}} S\right|^{2}\right]^{3 / 2}} \\
& +O\left(\varepsilon^{2 \alpha}\right)
\end{aligned}
$$

where we have used $\triangle_{\xi} u_{1}=0$ and $\triangle_{\eta} u_{2}=0$. 
and on $\Gamma_{2}^{\varepsilon}$

$$
\begin{aligned}
v_{0}= & (0, \cdots, 0,1), \\
v_{\varepsilon}= & \left(\nabla_{\eta^{\prime}} T, 1\right) \frac{1}{\left[1+\left|\nabla_{\eta^{\prime}} T\right|^{2}\right]^{1 / 2}} \\
& +\varepsilon^{\beta}\left(\nabla_{x^{\prime}} T,-\nabla_{x^{\prime}} T \cdot \nabla_{\eta^{\prime}} T\right) \frac{1}{\left[1+\left|\nabla_{\eta^{\prime}} T\right|^{2}\right]^{3 / 2}} \\
& +O\left(\varepsilon^{2 \beta}\right) .
\end{aligned}
$$

In view of (33)-(42), we obtain

$$
\begin{aligned}
& \left|\int_{\Omega_{\varepsilon}}\left(\varepsilon^{\alpha} \triangle_{x} u_{1}+\varepsilon^{\beta} \triangle_{x} u_{2}\right) v d x\right| \\
& \leq C \varepsilon^{\alpha} \int_{\Omega_{\varepsilon}} \exp \left(-\frac{\lambda x_{1}}{\varepsilon^{\alpha}}\right)|v| d x \\
& +C \varepsilon^{\beta} \int_{\Omega_{\varepsilon}} \exp \left(-\frac{\theta x_{1}}{\varepsilon^{\beta}}\right)|v| d x \leq C\left(\varepsilon^{3 \alpha / 2}\right. \\
& \left.+\varepsilon^{3 \beta / 2}\right)\|v\|_{H^{1}\left(\Omega_{0}\right)}, \\
& 2 \int_{\Omega_{\varepsilon}} \nabla_{x} \cdot \nabla_{\xi} u_{1} v d x=2 \varepsilon^{\alpha} \int_{\Gamma_{1}^{\varepsilon}} \nabla_{x} u_{1} \cdot v_{\varepsilon} v d \sigma_{1} \\
& -2 \varepsilon^{\alpha} \int_{\Omega_{\varepsilon}} \nabla_{x} u_{1} \cdot \nabla_{x} v d x \leq 2 \varepsilon^{\alpha} \int_{\Gamma_{1}^{\varepsilon}} \nabla_{x} u_{1} \cdot v_{\varepsilon} v d \sigma_{1} \\
& +C \varepsilon^{\alpha} \int_{\Omega_{\varepsilon}} \exp \left(-\lambda \xi_{1}\right)\left|\nabla_{x} v\right| d x \leq 2 \varepsilon^{\alpha} \int_{\Gamma_{1}^{\varepsilon}} \nabla_{x} u_{1} \\
& \cdot\left(\nabla_{\xi^{\prime}} S,-1\right) \frac{1}{\left[1+\left|\nabla_{\xi^{\prime}} S\right|^{2}\right]^{1 / 2}} v d \sigma_{1} \\
& +C \varepsilon^{3 \alpha / 2}\|v\|_{H^{1}\left(\Omega_{0}\right)}+2 \varepsilon^{2 \alpha} \int_{\Gamma_{1}^{\varepsilon}} \nabla_{x} u_{1} \\
& \cdot\left(\nabla_{x^{\prime}} S, \nabla_{x^{\prime}} S \cdot \nabla_{\xi^{\prime}} S\right) \frac{1}{\left[1+\left|\nabla_{\xi^{\prime}} S\right|^{2}\right]^{3 / 2}} v d \sigma_{1}
\end{aligned}
$$

and

$$
\begin{aligned}
& 2 \int_{\Omega_{\varepsilon}} \nabla_{x} \cdot \nabla_{\eta} u_{2} v d x \leq 2 \varepsilon^{\beta} \int_{\Gamma_{2}^{\varepsilon}} \nabla_{x} u_{2} \\
& \cdot\left(\nabla_{\eta^{\prime}} T, 1\right) \frac{1}{\left[1+\left|\nabla_{\eta^{\prime}} T\right|^{2}\right]^{1 / 2}} v d \sigma_{2} \\
& +C \varepsilon^{3 \beta / 2}\|v\|_{H^{1}\left(\Omega_{0}\right)}+2 \varepsilon^{2 \beta} \int_{\Gamma_{2}^{\varepsilon}} \nabla_{x} u_{2} \\
& \cdot\left(\nabla_{x^{\prime}} T,-\nabla_{x^{\prime}} T \cdot \nabla_{\eta^{\prime}} T\right) \frac{1}{\left[1+\left|\nabla_{\eta^{\prime}} T\right|^{2}\right]^{3 / 2}} v d \sigma_{2} .
\end{aligned}
$$

It follows from (38) that

$$
\begin{aligned}
\left.\varepsilon^{\alpha} \frac{\partial u_{1}}{\partial \nu_{\varepsilon}}\right|_{\Gamma_{1}^{\varepsilon}} & =\left.\left(\nabla_{\xi} u_{1} \cdot v_{\varepsilon}+\varepsilon^{\alpha} \nabla_{x} u_{1} \cdot v_{\varepsilon}\right)\right|_{\Gamma_{1}^{\varepsilon}} \\
& =\widetilde{A}_{1}+\widetilde{A}_{2}+O\left(\varepsilon^{2 \alpha}\right),
\end{aligned}
$$

where

$$
\begin{aligned}
\widetilde{A}_{1}= & \left(g-\frac{p}{P} G\right)-\nabla_{x^{\prime}} u_{0} \cdot \nabla_{\xi^{\prime}} S \frac{1}{\left[1+\left|\nabla_{\xi^{\prime}} S\right|^{2}\right]^{1 / 2}} \\
& +\frac{\partial u_{0}}{\partial x_{n}}\left(\frac{1}{\left[1+\left|\nabla_{\xi^{\prime}} S\right|^{2}\right]^{1 / 2}}-\frac{p}{P}\right) \text { and } \\
\widetilde{A}_{2}= & \varepsilon^{\alpha} \nabla_{x} u_{1} \cdot\left(\nabla_{\xi^{\prime}} S,-1\right) \frac{1}{\left[1+\left|\nabla_{\xi^{\prime}} S\right|^{2}\right]^{1 / 2}} .
\end{aligned}
$$

Combining all these terms in (77), we have proved that

$$
\begin{aligned}
\int_{\Gamma_{1}^{\varepsilon}} \varepsilon^{\alpha} \frac{\partial u_{1}}{\partial \nu_{\varepsilon}} v d \sigma_{1}= & \int_{\Gamma_{1}^{\varepsilon}} \widetilde{A}_{1} v d \sigma_{1}+\int_{\Gamma_{1}^{\varepsilon}} \widetilde{A}_{2} v d \sigma_{1} \\
& +O\left(\varepsilon^{2 \alpha}\right)\|v\|_{H^{1}\left(\Omega_{0}\right)} .
\end{aligned}
$$

A similar calculation yields the following result:

$$
\begin{aligned}
& \int_{\Gamma_{1}^{\varepsilon}}\left(\frac{p}{P} \frac{\partial u_{0}}{\partial v_{0}}-\frac{\partial u_{0}}{\partial \nu_{\varepsilon}}\right) v d \sigma_{1} \\
& =\int_{\Gamma_{1}^{\varepsilon}}-\frac{p}{P} \frac{\partial u_{0}}{\partial x_{n}} v d \sigma_{1}-\widetilde{A}+O\left(\varepsilon^{2 \alpha}\right)\|v\|_{H^{1}\left(\Omega_{0}\right)},
\end{aligned}
$$

where

$$
\begin{aligned}
\widetilde{A}= & \int_{\Gamma_{1}^{\varepsilon}} \frac{1}{\left[1+\left|\nabla_{\xi^{\prime}} S\right|^{2}\right]^{1 / 2}}\left(\nabla_{x^{\prime}} u_{0} \cdot \nabla_{\xi^{\prime}} S-\frac{\partial u_{0}}{\partial x_{n}}\right) \\
& +\varepsilon^{\alpha} \nabla u_{0} \\
& \cdot\left(\nabla_{x^{\prime}} S, \nabla_{x^{\prime}} S \cdot \nabla_{\xi^{\prime}} S\right) \frac{1}{\left[1+\left|\nabla_{\xi^{\prime}} S\right|^{2}\right]^{3 / 2}} v d \sigma_{1} .
\end{aligned}
$$

In the same way, it follows from (42) that

$$
\begin{aligned}
\left.\varepsilon^{\beta} \frac{\partial u_{2}}{\partial \nu_{\varepsilon}}\right|_{\Gamma_{2}^{\varepsilon}} & =\left.\left(\nabla_{\eta} u_{2} \cdot v_{\varepsilon}+\varepsilon^{\beta} \nabla_{x} u_{2} \cdot v_{\varepsilon}\right)\right|_{\Gamma_{2}^{\varepsilon}} \\
& =\widetilde{B}_{1}+\widetilde{B}_{2}+O\left(\varepsilon^{2 \beta}\right),
\end{aligned}
$$

where 


$$
\begin{aligned}
\widetilde{B}_{1}= & \left(h-\frac{q}{Q} H\right)-\nabla_{x^{\prime}} u_{0} \cdot \nabla_{\eta^{\prime}} T \frac{1}{\left[1+\left|\nabla_{\xi^{\prime}} T\right|^{2}\right]^{1 / 2}} \\
& +\frac{\partial u_{0}}{\partial x_{n}}\left(\frac{-1}{\left[1+\left|\nabla_{\eta^{\prime}} T\right|^{2}\right]^{1 / 2}}+\frac{q}{Q}\right) \text { and } \\
\widetilde{B}_{2}= & \varepsilon^{\beta} \nabla_{x} u_{2} \cdot\left(\nabla_{\eta^{\prime}} T, 1\right) \frac{1}{\left[1+\left|\nabla_{\eta^{\prime}} T\right|^{2}\right]^{1 / 2}} .
\end{aligned}
$$

Combining all these terms in (82), we have proved that

$$
\begin{aligned}
\int_{\Gamma_{2}^{\varepsilon}} \varepsilon^{\beta} \frac{\partial u_{2}}{\partial \nu_{\varepsilon}} v d \sigma_{2}= & \int_{\Gamma_{2}^{\varepsilon}} \widetilde{B}_{1} v d \sigma_{2}+\int_{\Gamma_{2}^{\varepsilon}} \widetilde{B}_{2} v d \sigma_{2} \\
& +O\left(\varepsilon^{2 \beta}\right)\|v\|_{H^{1}\left(\Omega_{0}\right)} .
\end{aligned}
$$

Similarly, we obtain the following result:

$$
\begin{aligned}
& \int_{\Gamma_{2}^{\varepsilon}}\left(\frac{q}{Q} \frac{\partial u_{0}}{\partial \nu_{0}}-\frac{\partial u_{0}}{\partial \nu_{\varepsilon}}\right) v d \sigma_{2} \\
& \quad=\int_{\Gamma_{2}^{\varepsilon}} \frac{q}{Q} \frac{\partial u_{0}}{\partial x_{n}} v d \sigma_{2}-\widetilde{B}+O\left(\varepsilon^{2 \beta}\right)\|v\|_{H^{1}\left(\Omega_{0}\right)},
\end{aligned}
$$

where

$$
\begin{aligned}
\widetilde{B} & =\int_{\Gamma_{2}^{\varepsilon}} \frac{1}{\left[1+\left|\nabla_{\eta^{\prime}} T\right|^{2}\right]^{1 / 2}}\left(\nabla_{x^{\prime}} u_{0} \cdot \nabla_{\eta^{\prime}} T+\frac{\partial u_{0}}{\partial x_{n}}\right) \\
& +\varepsilon^{\beta} \nabla u_{0} \\
& \cdot\left(\nabla_{x^{\prime}} T,-\nabla_{x^{\prime}} T \cdot \nabla_{\eta^{\prime}} T\right) \frac{1}{\left[1+\left|\nabla_{\eta^{\prime}} T\right|^{2}\right]^{3 / 2}} v d \sigma_{2} .
\end{aligned}
$$

Next, we shall introduce two new correctors so as to cancel lower power of $\varepsilon^{\alpha}$ and $\varepsilon^{\beta}$.

For any given function of the form $\tilde{g}$, suppose that $\widetilde{u}_{\varepsilon}$ is the solution of the following problem:

$$
\begin{aligned}
-\triangle \widetilde{u}_{\varepsilon} & =0 \quad \text { in } \Omega_{\varepsilon}, \\
\frac{\partial \widetilde{u}_{\varepsilon}}{\partial v_{\varepsilon}}+p\left(x^{\prime}, \frac{x^{\prime}}{\varepsilon^{\alpha}}\right) \widetilde{u}_{\varepsilon} & =\widetilde{g}\left(x^{\prime}, \frac{x^{\prime}}{\varepsilon^{\alpha}}\right) \quad \text { on } \Gamma_{1}^{\varepsilon}, \\
\widetilde{u}_{\varepsilon} & =0 \text { on } \partial \Omega_{\varepsilon} \backslash \Gamma_{1}^{\varepsilon} .
\end{aligned}
$$

Meanwhile, we assume that $\phi_{1}$ is the solution of the homogenized problem:

$$
\begin{aligned}
-\Delta \phi_{1} & =0 \quad \text { in } \Omega_{0}, \\
\frac{\partial \phi_{1}}{\partial \nu_{0}}+P\left(x^{\prime}\right) \phi_{1} & =\widetilde{G}\left(x^{\prime}\right) \quad \text { on } \Gamma_{1}, \\
\phi_{1} & =0 \quad \text { on } \partial \Omega_{0} \backslash \Gamma_{1},
\end{aligned}
$$

where $P\left(x^{\prime}\right)$ and $\Gamma_{1}^{\varepsilon}$ are defined in (8) and (2),

$$
\begin{aligned}
& \widetilde{G}\left(x^{\prime}\right) \\
& \quad=\int_{0}^{1} \cdots \int_{0}^{1}\left[1+\left|\nabla_{\xi^{\prime}} S\left(x^{\prime}, \xi^{\prime}\right)\right|^{2}\right]^{1 / 2} \tilde{g}\left(x^{\prime}, \xi^{\prime}\right) d \xi^{\prime} .
\end{aligned}
$$

Clearly, it is a special case of problem (1).

We may invoke Theorem 8 to conclude that

$$
\left\|\tilde{u}_{\varepsilon}-\phi_{1}-\varepsilon^{\alpha} \widetilde{u}_{1}\right\|_{H^{1}\left(\Omega_{\varepsilon}\right)} \leq C \varepsilon^{\alpha},
$$

where $\widetilde{u}_{1}$ follows essentially the same steps as Section 4 , with the obvious modifications.

It follows that

$$
\begin{aligned}
B\left(\varepsilon^{\alpha} \phi_{1}, v\right)= & B\left(\varepsilon^{\alpha}\left(\phi_{1}-\widetilde{u}_{\varepsilon}+\varepsilon^{\alpha} \widetilde{u}_{1}\right), v\right) \\
& +B\left(\varepsilon^{\alpha} \widetilde{u}_{\varepsilon}-\varepsilon^{2 \alpha} \widetilde{u}_{1}, v\right) \\
= & B\left(\varepsilon^{\alpha} \widetilde{u}_{\varepsilon}, v\right)+O\left(\varepsilon^{2 \alpha}\right)\|v\|_{H^{1}\left(\Omega_{0}\right)} .
\end{aligned}
$$

In a similar way, for any given function of the form $\tilde{h}$, suppose that $\widehat{u}_{\varepsilon}$ is the solution of the following problem:

$$
\begin{aligned}
-\Delta \widehat{u}_{\varepsilon} & =0 \quad \text { in } \Omega_{\varepsilon}, \\
\frac{\partial \widehat{u}_{\varepsilon}}{\partial \nu_{\varepsilon}}+q\left(x^{\prime}, \frac{x^{\prime}}{\varepsilon^{\beta}}\right) \widehat{u}_{\varepsilon} & =\widehat{h}\left(x^{\prime}, \frac{x^{\prime}}{\varepsilon^{\beta}}\right) \quad \text { on } \Gamma_{2}^{\varepsilon}, \\
\widehat{u}_{\varepsilon} & =0 \quad \text { on } \partial \Omega_{\varepsilon} \backslash \Gamma_{2}^{\varepsilon} .
\end{aligned}
$$

Analogously to $\phi_{1}$, let $\phi_{2}$ be the solution of the homogenized problem

$$
\begin{aligned}
-\triangle \phi_{2} & =0 \quad \text { in } \Omega_{0}, \\
\frac{\partial \phi_{2}}{\partial \nu_{0}}+Q\left(x^{\prime}\right) \phi_{2} & =\widehat{H}\left(x^{\prime}\right) \quad \text { on } \Gamma_{2}, \\
\phi_{2} & =0 \quad \text { on } \partial \Omega_{0} \backslash \Gamma_{2},
\end{aligned}
$$

where $Q\left(x^{\prime}\right)$ and $\Gamma_{2}^{\varepsilon}$ are defined in (8) and (3):

$$
\begin{aligned}
\widehat{H} & \left(x^{\prime}\right) \\
& =\int_{0}^{1} \cdots \int_{0}^{1}\left[1+\left|\nabla_{\eta^{\prime}} T\left(x^{\prime}, \eta^{\prime}\right)\right|^{2}\right]^{1 / 2} \widehat{h}\left(x^{\prime}, \eta^{\prime}\right) d \eta^{\prime} .
\end{aligned}
$$

In view of Theorem 8 ,

$$
\left\|\widetilde{u}_{\varepsilon}-\phi_{2}-\varepsilon^{\beta} \widehat{u}_{2}\right\|_{H^{1}\left(\Omega_{\varepsilon}\right)} \leq C \varepsilon^{\beta},
$$

where $\widehat{u}_{2}$ follows essentially the same steps as Section 4 , with the obvious modifications.

It follows that

$$
B\left(\varepsilon^{\beta} \phi_{2}, v\right)=B\left(\varepsilon^{\beta} \widehat{u}_{\varepsilon}, v\right)+O\left(\varepsilon^{2 \beta}\right)\|v\|_{H^{1}\left(\Omega_{0}\right)} .
$$

We now write

$$
\begin{aligned}
& B\left(u_{\varepsilon}-u_{0}-\varepsilon^{\alpha} u_{1}-\varepsilon^{\beta} u_{2}+\varepsilon^{\alpha} \phi_{1}+\varepsilon^{\beta} \phi_{2}, v\right) \\
& =\varepsilon^{\alpha} \int_{\Gamma_{1}} \tilde{g} v d x^{\prime}+\varepsilon^{\beta} \int_{\Gamma_{2}} \widehat{h} v d x^{\prime}-\varepsilon^{\alpha} \sum_{i=1}^{3} I_{i}-\varepsilon^{\beta} \sum_{i=1}^{3} L_{i} \\
& \quad+O\left(\varepsilon^{2 \alpha}+\varepsilon^{2 \beta}\right)\|v\|_{H^{1}\left(\Omega_{0}\right)},
\end{aligned}
$$

where 


$$
\begin{aligned}
I_{1} & =\int_{\Gamma_{1}} \nabla u_{0} \cdot\left(\nabla_{x^{\prime}} S, \nabla_{x^{\prime}} S \cdot \nabla_{\xi^{\prime}} S\right) v d x^{\prime}, \\
I_{2} & =\left.\int_{\Gamma_{1}^{\varepsilon}} \frac{p}{P} S \frac{\partial}{\partial x_{n}}\left(\frac{\partial u_{0}}{\partial v_{0}}+P u_{0}\right)\right|_{\Gamma_{1}} v d \sigma_{1}, \\
I_{3} & =-\int_{\Gamma_{1}} \nabla_{x} u_{1} \cdot\left(\nabla_{x^{\prime}} S,-1\right) v d x^{\prime}, \text { and } \\
L_{1} & =\int_{\Gamma_{2}} \nabla u_{0} \cdot\left(\nabla_{x^{\prime}} T,-\nabla_{x^{\prime}} T \cdot \nabla_{\eta^{\prime}} T\right) v d x^{\prime}, \\
L_{2} & =-\left.\int_{\Gamma_{2}^{\varepsilon}} \frac{q}{Q} T \frac{\partial}{\partial x_{n}}\left(\frac{\partial u_{0}}{\partial v_{0}}+Q u_{0}\right)\right|_{\Gamma_{2}} v d \sigma_{2}, \\
L_{3} & =-\int_{\Gamma_{2}} \nabla_{x} u_{2} \cdot\left(\nabla_{x^{\prime}} T, 1\right) v d x^{\prime} .
\end{aligned}
$$

Then we just need to take that

$$
\begin{aligned}
& \tilde{g}=\sum_{i=1}^{3} \widetilde{I}_{i} \text { and } \\
& \widehat{h}=\sum_{i=1}^{3} \widehat{L}_{i},
\end{aligned}
$$

where

$$
\begin{gathered}
\widetilde{I}_{1}=\nabla u_{0} \cdot\left(\nabla_{x^{\prime}} S, \nabla_{x^{\prime}} S \cdot \nabla_{\xi^{\prime}} S\right), \\
\widetilde{I}_{2}=\left.\frac{p}{P} S \frac{\partial}{\partial x_{n}}\left(\frac{\partial u_{0}}{\partial v_{0}}+P u_{0}\right)\right|_{\Gamma_{1}}\left[1+\left|\nabla_{\xi^{\prime}} S\left(x^{\prime}, \xi^{\prime}\right)\right|^{2}\right]^{1 / 2}, \\
\widetilde{I}_{3}=-\nabla_{x} u_{1} \cdot\left(\nabla_{x^{\prime}} S,-1\right) . \\
\widehat{L}_{1}=\nabla u_{0} \cdot\left(\nabla_{x^{\prime}} T,-\nabla_{x^{\prime}} T \cdot \nabla_{\eta^{\prime}} T\right), \\
\widehat{L}_{2}=-\left.\frac{q}{Q} T \frac{\partial}{\partial x_{n}}\left(\frac{\partial u_{0}}{\partial v_{0}}+Q u_{0}\right)\right|_{\Gamma_{2}}\left[1+\left|\nabla_{\eta^{\prime}} T\left(x^{\prime}, \eta^{\prime}\right)\right|^{2}\right]^{1 / 2}, \\
\widehat{L}_{3}=-\nabla_{x} u_{2} \cdot\left(\nabla_{x^{\prime}} T, 1\right) .
\end{gathered}
$$

Combining all these terms (70)-(97) and choosing $v=$ $u_{\varepsilon}-u_{0}-\varepsilon^{\alpha}\left(u_{1}-\left.u_{1}\right|_{x_{i}=1}\right)-\varepsilon^{\beta}\left(u_{2}-\left.u_{2}\right|_{x_{i}=1}\right)+\varepsilon^{\alpha} \phi_{1}+\varepsilon^{\beta} \phi_{2}$, the proof is completed.

\section{Data Availability}

No data were used to support this study.

\section{Conflicts of Interest}

The authors declare that they have no conflicts of interest.

\section{Acknowledgments}

The authors would like to express sincere thanks to professor D. S. Li for his valuable suggestions and useful discussions. It is also a pleasure to thank professor $\mathrm{B}$. Hu for stimulating The authors' interest to this problem. This work was supported by NSFC (11626239), the China Scholarship Council (201708410483), and Foundation of Education Department of Henan Province (18A110037).

\section{References}

[1] Y. Achdou and O. Pironneau, "Domain decomposition and wall laws," Comptes Rendus de Académie des Sciences - Series, vol. 320, no. 5, pp. 541-547, 1995.

[2] Y. Achdou and O. Pironneau, "A 2nd order condition for flow over rough walls," in Proceedings of the International Conference on Nonlinear Differential Equations and Applications, Shrikant ED, Bangalore, India, 1996.

[3] G. Buttazzo and R. V. Kohn, "Reinforcement by a thin layer with oscillating thickness," Applied Mathematics \& Optimization, vol. 16, no. 3, pp. 247-261, 1987.

[4] T. Abboud and H. Ammari, "Diffraction at a curved grating: TM and TE cases, homogenization," Journal of Mathematical Analysis and Applications, vol. 202, no. 3, pp. 995-1026, 1996.

[5] B. Engquist and J. C. Nédélec, Effective boundary conditions for accoustic ans electro-magnetic scaterring in thin layers 278, CMAP École Polytechnique, 1993.

[6] G. Allaire and G. Bal, "Homogenization of the criticality spectral equation in neutron transport," M2AN: Mathematical Modelling and Numerical Analysis, vol. 33, no. 4, pp. 721-746, 1999.

[7] G. Bal, "First-order corrector for the homogenization of the criticality eigenvalue problem in the even parity formulation of the neutron transport," SIAM Journal on Mathematical Analysis, vol. 30, no. 6, pp. 1208-1240, 1999.

[8] A. Bensoussan, J.-L. Lions, and G. C. Papanicolaou, "Boundary layers and homogenization of transport processes," Publications of the Research Institute for Mathematical Sciences, vol. 15, no. 1, pp. 53-157, 1979.

[9] I. Babuas'ka, "Solution of interface problems by homogenization," SIAM Journal on Mathematical Analysis, vol. 7, no. 5, pp. 603-634, 1976.

[10] A. Bourgeat and E. Marusi-Paloka, "Nonlinear effects for flow in periodically constricted channel caused by high injection rate," Mathematical Models and Methods in Applied Sciences, vol. 8, no. 3, pp. 379-405, 1998.

[11] W. Jäger and A. Mikelic, "On the boundary conditions at the contact interface between a porous medium and a free fluid," Annali della Scuola Normale Superiore di Pisa-Classe di Scienze, vol. 23, pp. 403-465, 1996.

[12] A. Friedman, B. Hu, and Y. Liu, "A boundary value problem for the Poisson equation with multi-scale oscillating boundary," Journal of Differential Equations, vol. 137, no. 1, pp. 54-93, 1997.

[13] A. G. Belyaev, "Averaging the third boundary value problem for the Poisson equation in a domain with a rapidly oscillating boundary," Vestnik Moskovskogo Universiteta. Seriya 1. Matematika. Mekhanika, no. 6, pp. 63-66, 1988.

[14] A. G. Belyaev, On singular perturbations of boundary problems [Ph.D. thesis], Moscow State University, Moscow, Russia, 1990 (Russian).

[15] G. A. Chechkin, A. Friedman, and A. L. Piatnitski, "The boundary-value problem in domains with very rapidly oscillating boundary," Journal of Mathematical Analysis and Applications, vol. 231, no. 1, pp. 213-234, 1999.

[16] D. Gérard and N. Masmoudi, "Homogenization and boundary layers," Acta Mathematica, vol. 209, no. 1, pp. 133-178, 2012.

[17] H. Aleksanyan, H. Shahgholian, and P. Sjolin, "Applications of Fourier analysis in homogenization of Dirichlet problem I. Pointwise estimates," Journal of Differential Equations, vol. 254, no. 6, pp. 2626-2637, 2013. 
[18] J. M. Arrieta and M. C. Pereira, "Homogenization in a thin domain with an oscillatory boundary," Journal de Mathématiques Pures et Apliquées, vol. 96, pp. 29-57, 2011.

[19] J. M. Arrieta and M. C. Pereira, "The Neumann problem in thin domains with very highly oscillatory boundaries," Journal of Mathematical Analysis and Applications, vol. 404, no. 1, pp. 86-104, 2013.

[20] A. Bensoussan, J.-L. Lions, and G. Papanicolaou, Asymptotic Analysis for Periodic Structures, 1978.

[21] U. Hornung, Homogenization and Porous Media, Springer, New York, NY, USA, 1997.

[22] A. Braides and A. Defranceschi, Homogenization of multiple integrals, Clarenoon, Oxford, 1998.

[23] D. Cioranescu and J. Saint Jean Paulin, Homogenization of Reticulated Structures, vol. 136 of Applied Mathematical Sciences, Springer, New York, NY, USA, 1999.

[24] D. Cioranescu and P. Donato, An Introduction to Homogenization, vol. 17 of Oxford Lecture Series in Mathematics and its Applications, Oxford University Press, New York, NY, USA, 1999.

[25] G. Allaire, Shape Optimization by the Homogenization Method, Springer-Verlag, New York, NY, USA, 2002.

[26] L. I. Manevitch, I. V. Andrianov, and V. G. Oshmyan, Mechanics of Periodically Heterogeneous Structures, Springer-Verlag, New York, NY, USA, 2002. 


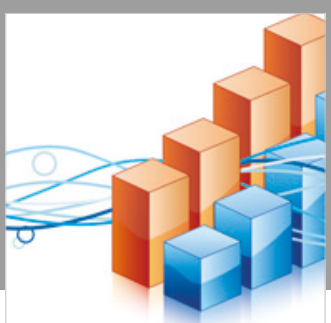

Advances in

Operations Research

\section{-n-m}
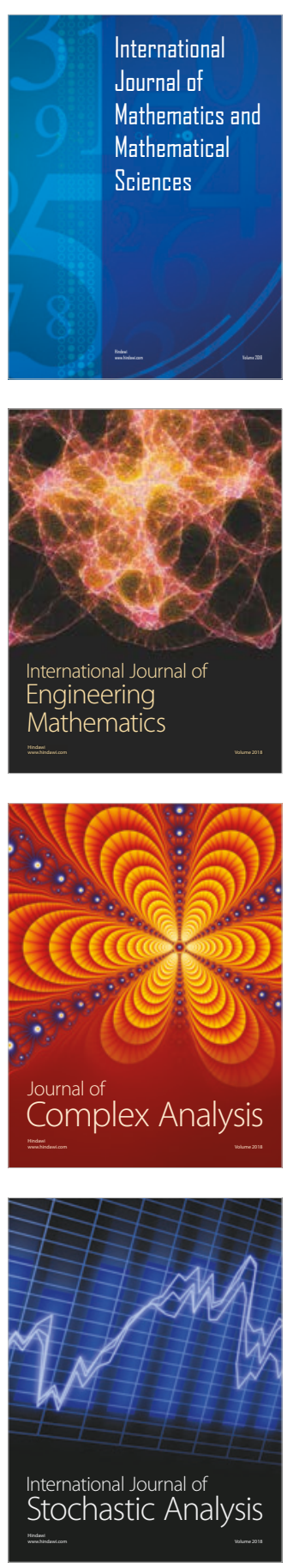
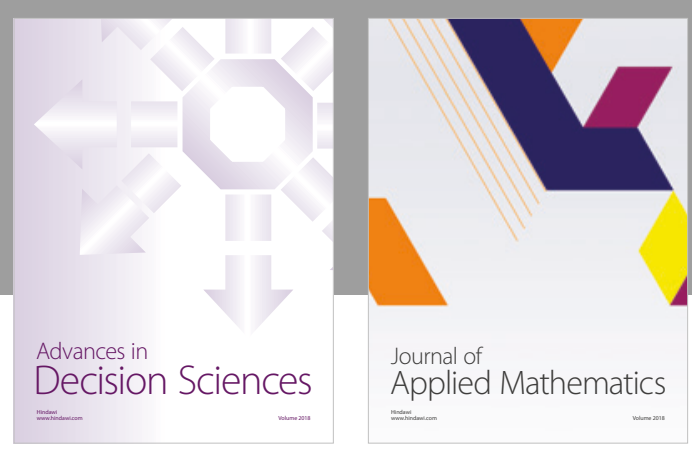

Journal of

Applied Mathematics
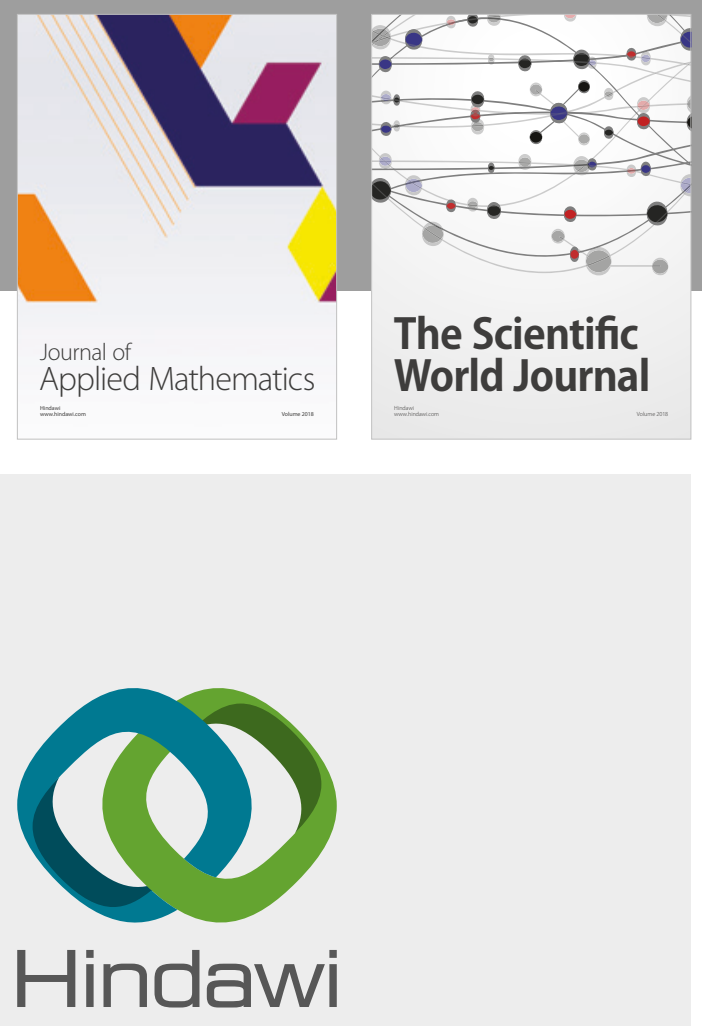

Submit your manuscripts at

www.hindawi.com

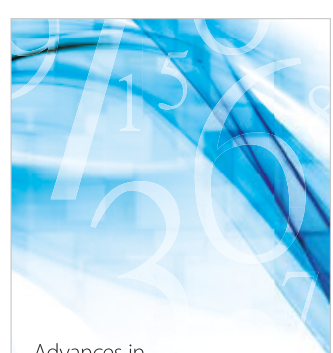

Advances in
Numerical Analysis
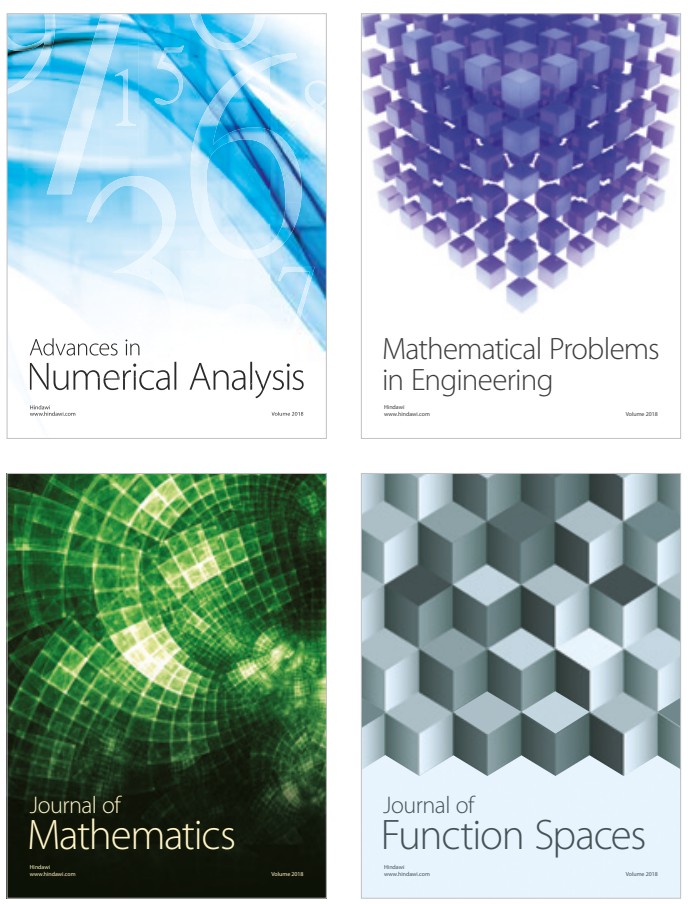

Mathematical Problems in Engineering

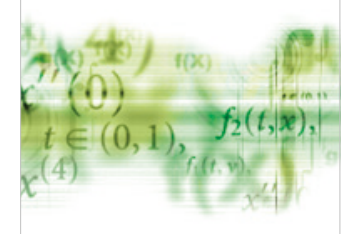

International Journal of

Differential Equations

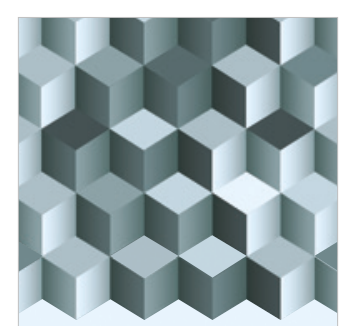

Journal of

Function Spaces
The Scientific

World Journal

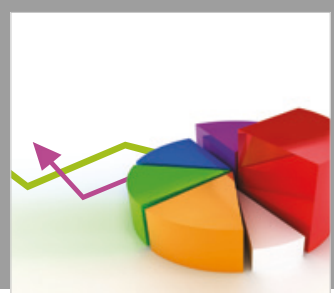

Journal of

Probability and Statistics
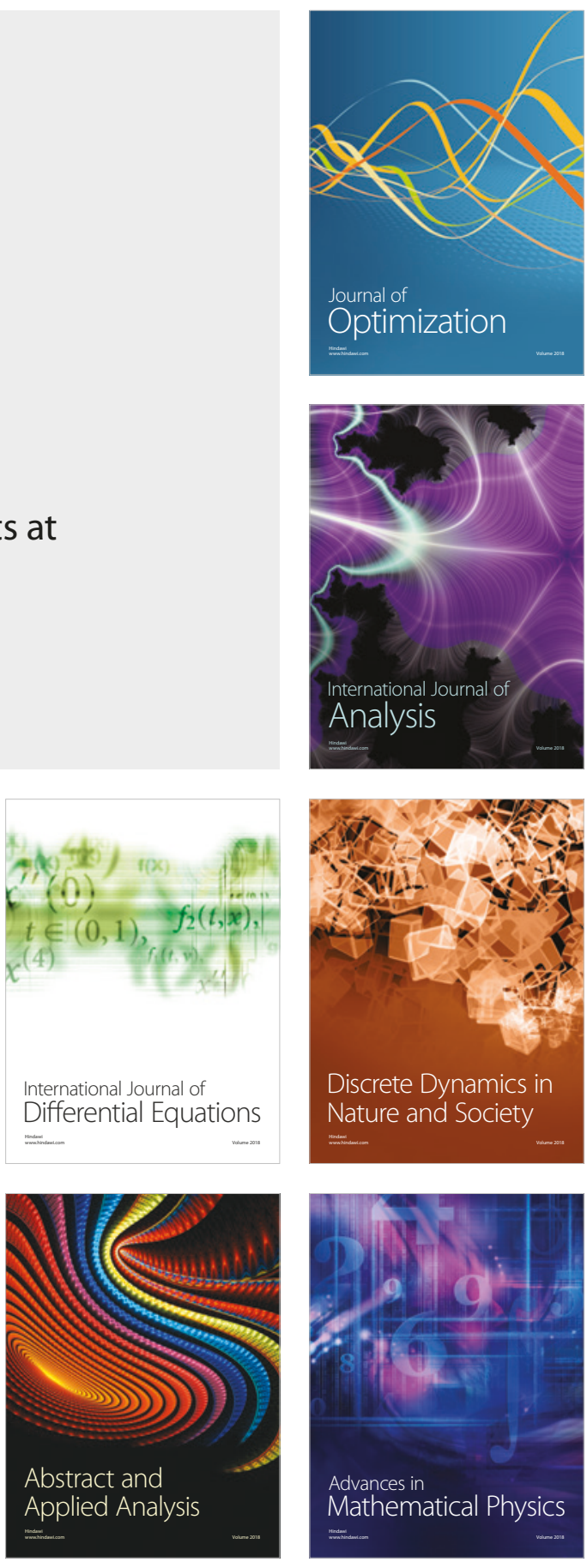